\title{
An approach to Quillen's conjecture via centralisers of simple groups
}

\author{
Kevin Iván Piterman \\ Departamento de Matemática, IMAS-CONICET, FCEyN, Universidad de Buenos Aires, Buenos Aires, Argentina; \\ E-mail: kpiterman@dm.uba.ar.
}

Received: 3 April 2021; Revised: 14 April 2021; Accepted: 4 May 2021

2020 Mathematics Subject Classification: Primary - 06A11, 20D30, 20J05; Secondary - 05E18, 20D05, 20D25

\begin{abstract}
For any given subgroup $H$ of a finite group $G$, the Quillen poset $\mathcal{A}_{p}(G)$ of nontrivial elementary abelian $p$-subgroups is obtained from $\mathcal{A}_{p}(H)$ by attaching elements via their centralisers in $H$. We exploit this idea to study Quillen's conjecture, which asserts that if $\mathcal{A}_{p}(G)$ is contractible then $G$ has a nontrivial normal $p$-subgroup. We prove that the original conjecture is equivalent to the $\mathbb{Z}$-acyclic version of the conjecture (obtained by replacing 'contractible' by ' $\mathbb{Z}$-acyclic'). We also work with the $\mathbb{Q}$-acyclic (strong) version of the conjecture, reducing its study to extensions of direct products of simple groups of $p$-rank at least 2. This allows us to extend results of Aschbacher and Smith and to establish the strong conjecture for groups of $p$-rank at most 4.
\end{abstract}

\section{Introduction}

Given a finite group $G$ and a prime number $p$, let $\mathcal{A}_{p}(G)$ be the Quillen poset of nontrivial elementary abelian $p$-subgroups of $G$. We can study the homotopical properties of $\mathcal{A}_{p}(G)$ by means of its order complex. In [16], Quillen proved that $\mathcal{A}_{p}(G)$ is contractible if $G$ has a nontrivial normal $p$-subgroup. He also conjectured the converse, giving rise to the well-known Quillen conjecture. That is, if $\mathcal{A}_{p}(G)$ is contractible, then $G$ has a nontrivial normal $p$-subgroup; or equivalently, if $G$ has no nontrivial normal $p$-subgroup, then $\mathcal{A}_{p}(G)$ is not contractible. This conjecture has been widely studied during the past decades and various cases have been proved, but the full conjecture remains open in general.

In this article, we consider the following versions of the conjecture. Let $O_{p}(G)$ be the largest normal $p$-subgroup of $G$, and $\tilde{H}_{*}(X, R)$ the reduced homology of a finite poset $X$ (which is the homology of its order complex $\mathcal{K}(X)$ ), with coefficients in the ring $R$.

(QC) If $O_{p}(G)=1$, then $\mathcal{A}_{p}(G)$ is not contractible.

$\left(R\right.$-QC) If $O_{p}(G)=1$, then $\tilde{H}_{*}\left(\mathcal{A}_{p}(G), R\right) \neq 0$.

We will usually take $R=\mathbb{Z}$ or $\mathbb{Q}$. Hence we have the following:

$$
\begin{aligned}
& (\mathbb{Z}-\mathrm{QC}) \quad \text { If } O_{p}(G)=1 \text {, then } \tilde{H}_{*}\left(\mathcal{A}_{p}(G), \mathbb{Z}\right) \neq 0 . \\
& (\mathbb{Q}-\mathrm{QC}) \quad \text { If } O_{p}(G)=1 \text {, then } \tilde{H}_{*}\left(\mathcal{A}_{p}(G), \mathbb{Q}\right) \neq 0 .
\end{aligned}
$$

Note that $(\mathbb{Q}-Q C)$ implies $(\mathbb{Z}-Q C)$, which implies the original conjecture $(\mathrm{QC})$.

The most important advances on the conjecture were achieved on the stronger version $(\mathbb{Q}-Q C)$. Quillen established $(\mathbb{Q}-\mathrm{QC})$ for solvable groups, groups of $p$-rank at most 2 and some families of groups

(C) The Author(s), 2021. Published by Cambridge University Press. This is an Open Access article, distributed under the terms of the Creative Commons Attribution licence (http://creativecommons.org/licenses/by/4.0/), which permits unrestricted re-use, distribution, and reproduction in any medium, provided the original work is properly cited. 
of Lie type [16]. Later, various authors dealt with the $p$-solvable case (see [1, 7] and [20, Chapter 8]), and in [4] Aschbacher and Kleidman showed (Q-QC) for almost-simple groups. In [5] Aschbacher and Smith proved that a group $G$ satisfies ( $Q-Q C)$ if $p>5$, and whenever $G$ has a unitary component $\mathrm{U}_{n}(q)$ with $q \equiv-1(\bmod p)$ and $q$ odd, then $(\mathcal{D D})_{p}$ holds for all $p$-extensions of $\mathrm{U}_{m}\left(q^{p^{e}}\right)$ with $m \leq n$ and $e \in \mathbb{Z}$ (see Definition 3.2). In a joint work with Sadofschi Costa and Viruel [14], we proved new cases of the conjecture not included in the previously mentioned results. We worked with the integer version of the conjecture (ZZ-QC) and proved that it holds if $\mathcal{K}\left(\mathcal{S}_{p}(G)\right)$ is homotopy-equivalent to a 2-dimensional and $G$-invariant subcomplex. Recall that $\mathcal{S}_{p}(G)$ is the Brown poset of nontrivial $p$-subgroups of $G$ and that $\mathcal{A}_{p}(G) \hookrightarrow \mathcal{S}_{p}(G)$ is a homotopy equivalence [16, Proposition 2.1]. In particular, the integer version holds for groups of $p$-rank at most 3. Recall that the $p$-rank of $G$ is the dimension of $\mathcal{K}\left(\mathcal{A}_{p}(G)\right)$ plus one.

Further applications and results concerning the homotopy type of the $p$-subgroup complexes can be found in $[6,9,15,20]$. In [10, Equation (1.4)], the authors considered a version of the conjecture even stronger than the rational one (Q-QC): if $O_{p}(G)=1$, then the Euler characteristic of $\mathcal{A}_{p}(G)$ is not 1 . We will not work with this version.

In this article, we approach the study of Quillen's conjecture via examination of the centralisers of the elementary abelian $p$-subgroups on suitable subgroups. Roughly, if $H$ is a nontrivial subgroup of $G$, then $\mathcal{A}_{p}(G)$ can be obtained first by passing from $\mathcal{A}_{p}(H)$ to the homotopy-equivalent subposet $\mathcal{N}(H)$, consisting of members $E \in \mathcal{A}_{p}(G)$ with $E \cap H \neq 1$, and then from $\mathcal{N}(H)$ to $\mathcal{A}_{p}(G)$ by attaching the remaining subgroups along their links in $\mathcal{N}(H)$. If $E \in \mathcal{A}_{p}(G)$ and $E \cap H=1$, its link in $\mathcal{N}(H)$ is $\mathcal{A}_{p}\left(C_{H}(E)\right)$, where $C_{H}(E)$ is the centraliser of $E$ in $H$. We can understand the homotopy type of $\mathcal{A}_{p}(G)$ from that of $\mathcal{A}_{p}(H)$ and the structure of these centralisers. In some cases, we extract points $E \in \mathcal{A}_{p}(G)$ with contractible link in $\mathcal{N}(H)$, and this is guaranteed precisely when $O_{p}\left(C_{H}(E)\right) \neq 1$. In this way, we can work with smaller subposets and apply inductive arguments. This approach has its roots in our previous work with Minian on the fundamental group of these complexes [12]. The poset $\mathcal{N}(H)$ was also considered by Segev and Webb $[18,19]$. In this article we put more emphasis on the attachment process, the behaviour of these centralisers as links and the extraction of points. This viewpoint seems to have been barely exploited, and we hope that the techniques and results of this article can shed more light on future methods for studying the topology of the $p$-subgroup posets and consequences beyond Quillen's conjecture.

We will study $(\mathbb{Z}-\mathrm{QC})$ and $(\mathbb{Q}-\mathrm{QC})$ by using this idea and working under the following inductive assumption. Let $R=\mathbb{Z}$ or $\mathbb{Q}$.

$(\mathrm{H} 1)_{R} \quad$ Proper subgroups and proper central quotients of $G$ satisfy $(R-\mathrm{QC})$.

By a 'proper central quotient of $G$ ', we mean a quotient of $G$ by a nontrivial central subgroup $Z \leq Z(G)$ (here $Z(G)$ denotes the center of $G)$. The hypothesis of the central quotients is motivated by the fact that if $Z \leq Z(G)$ is a $p^{\prime}$-group, then $\mathcal{A}_{p}(G)$ is naturally isomorphic to $\mathcal{A}_{p}(G / Z)$ (see Proposition 2.4).

The inductive assumption $(\mathrm{H} 1)_{R}$ is valid in the context of a counterexample of minimal order to the conjecture. That is, if $H$ satisfies $(R-\mathrm{QC})$ for all $|H|<|G|$, then $(\mathrm{H} 1)_{R}$ holds for $G$. Therefore, we may replace the content of $(\mathrm{H} 1)_{R}$ by this stronger inductive requirement.

The following theorem summarises some of the main results of this article. It shows $(R$-QC) under $(\mathrm{H} 1)_{R}$ and some extra hypothesis on the group. We follow the notation of [5, 8] for the simple groups.

Theorem 1. Let $G$ be a finite group and p a prime number. Let $R=\mathbb{Z}$ or $\mathbb{Q}$. Suppose that $(H 1)_{R}$ holds for $G$ and that one of the following holds:

1. $O_{p^{\prime}}(G) \neq 1$.

2. $\mathcal{A}_{p}(G)$ is not simply connected.

3. $p=3$ and $G$ has a component $L$ such that $L / Z(L) \cong \mathrm{U}_{3}\left(2^{3}\right)$.

4. $G$ has a component $L$ such that $L / Z(L)$ has p-rank 1 .

Then $G$ satisfies $(R-Q C)$. 
In particular, a counterexample of minimal order $G$ to $(R-Q C)$ fails conditions $1-4$, and hence it is an extension of a direct product of nonabelian simple groups of p-rank at least 2.

Our theorem has no restriction on the prime $p$ (except for condition 3 ) and is stated for both versions $(\mathbb{Z}-\mathrm{QC})$ and $(\mathbb{Q}-\mathrm{QC})$ of the conjecture. For example, condition 1 is a generalisation to every prime $p$ of the analogous result [5, Proposition 1.6] stated for $p>5$, and in our proof we use the classification of simple groups to a much lesser extent. Condition 2 of the theorem is based on our previous work on the fundamental group [11]. The more technical hypotheses of conditions 3 and 4 are focussed on extending [5, Main Theorem] to every odd prime $p$.

We describe now some consequences of Theorem 1.

First, it allows us to conclude that the original conjecture (QC) and the integer-homology version $(\mathbb{Z}-\mathrm{QC})$ are equivalent for every prime $p$.

Theorem 2. The original Quillen's conjecture and the integer Quillen's conjecture $(\mathbb{Z}-Q C)$ are equivalent. That is, $(Q C)$ holds for all finite groups if and only if $(\mathbb{Z}-Q C)$ holds for all finite groups.

This result strongly depends on the integer version of Theorem 1(1) and (2). It does not follow from [5, Proposition 1.6], which is stated for rational homology and $p>5$.

On the other hand, Theorem 1(3) and (4) allow us to handle the groups containing a component isomorphic to $\mathrm{L}_{2}\left(2^{3}\right)(p=3), \mathrm{U}_{3}\left(2^{3}\right)(p=3)$ or $\mathrm{Sz}\left(2^{5}\right)(p=5)$. Aschbacher and Smith excluded the groups containing these components during their analysis of the conjecture for odd $p$, mainly because the centralisers of their field automorphisms of order $p$ have nontrivial normal $p$-subgroups (see section 5 for a more detailed discussion). Nevertheless, our theorem shows that we can suppose that $G$ does not contain these components if we are aiming to prove Quillen's conjecture. This allows us to extend the main result of Aschbacher and Smith to $p=5$.

Corollary 3. [5, Main Theorem] also holds for $p=5$.

The extension of [5, Main Theorem] to $p=3$ is not immediate, since its proof depends on [5, Theorem 5.3], which is stated for $p \geq 5$.

Finally, we combine Theorem 1 with the classification of the simple groups of low $p$-rank, the structure of their centralisers and the classification of groups with a strongly $p$-embedded subgroup (i.e., with disconnected Quillen's complex) to yield the $p$-rank 4 case of the conjecture.

Theorem 4. If $G$ has $p$-rank at most 4 , then it satisfies $(\mathbb{Q}-Q C)$.

Theorem 1 follows from Theorems 4.1, 5.1 and 6.1, Corollary 4.4 and Remark 2.1. The proof of Theorem 2 is given in section 4, and the proof of Corollary 3 can be found after the statement of Theorem 5.1. Finally, Theorem 4 is proved in section 7. We also include an appendix containing basic properties of the almost-simple groups with a strongly $p$-embedded subgroup.

\section{Preliminary results}

In this section we establish the main definitions and tools that we will use throughout the paper. We refer to [3] for more details on finite group theory.

All the groups considered here are finite. By a simple group we mean a nonabelian simple group. We adopt the conventions of [8] for the names of the simple groups and their automorphisms. We denote by $C_{n}, D_{n}, \mathbb{S}_{n}$ and $\mathbb{A}_{n}$ the cyclic group of order $n$, the dihedral group of order $n$, the symmetric group on $n$ letters and the alternating group on $n$ letters, respectively.

Let $G$ be a finite group and $p$ a prime number. Denote by $Z(G)$ the center of $G$. Let $O_{p}(G)$ be the largest normal $p$-subgroup of $G$ and $O_{p^{\prime}}(G)$ be the largest normal $p^{\prime}$-subgroup of $G$. The Fitting subgroup $F(G)$ of $G$ is the largest normal nilpotent subgroup of $G$, and it is the direct product of the subgroups $O_{q}(G)$, for $q$ prime dividing the order of $G$. For a fixed prime $p$, define

\footnotetext{
${ }^{1}$ In a forthcoming work based on the results of this article, we also extend [5, Main Theorem] to $p=3$, and hence to every odd prime.
} 
$\Omega_{1}(G):=\left\langle x \in G: x^{p}=1\right\rangle$. The $p$-rank of $G$ is

$$
m_{p}(G):=1+\operatorname{dim} \mathcal{K}\left(\mathcal{A}_{p}(G)\right)=\max \left\{\log _{p}(|A|): A \in \mathcal{A}_{p}(G) \cup\{1\}\right\} .
$$

If $H, K \leq G$ are subgroups of $G$, then $N_{H}(K)$ denotes the normaliser of $K$ in $H$ and $C_{H}(K)$ the centraliser of $K$ in $H$. Denote by $[H, K]$ the subgroup generated by the commutators between elements of $H$ and $K$. If $g \in G$, write $H^{g}=g^{-1} H g$.

Recall that $E(G)$, the layer of $G$, is the (central) product of the components of $G$, and that the generalised Fitting subgroup $F^{*}(G)$ is the central product of $E(G)$ and $F(G)$. We refer to [3, Chapter 11] for the main properties of these subgroups.

Let $\operatorname{Out}(H)=\operatorname{Aut}(H) / \operatorname{Inn}(H)$ denote the group of outer automorphisms of $H$. If $H$ is a group of Lie type, $\operatorname{Inn} \operatorname{diag}(H)$ denotes the subgroup of inner-diagonal automorphisms of $H$, and Out $\operatorname{diag}(H)=$ $\operatorname{Inn} \operatorname{diag}(H) / \operatorname{Inn}(H)$.

Remark 2.1. If $O_{p}(G)=1=O_{p^{\prime}}(G)$, then $F(G) \leq O_{p}(G) O_{p^{\prime}}(G)=1$ and $F^{*}(G)=E(G)$ has trivial center. Therefore, $F^{*}(G)=L_{1} \cdots L_{n}$ is the direct product of the components $\left\{L_{1}, \ldots, L_{n}\right\}$ of $G$, which are nonabelian simple groups of order divisible by $p$. Since $F^{*}(G)$ is self-centralising, $F^{*}(G) \leq G \leq \operatorname{Aut}\left(F^{*}(G)\right)$. Moreover, $\operatorname{Aut}\left(F^{*}(G)\right)$ can be easily described by using the facts that if $L$ is a simple group, then $\operatorname{Aut}\left(L^{n}\right) \cong \operatorname{Aut}(L)<\mathbb{S}_{n}$ and that $\operatorname{Aut}(L \times K) \cong \operatorname{Aut}(L) \times \operatorname{Aut}(K)$ if $L$ and $K$ are nonisomorphic simple groups.

If $X$ is a finite poset, we can study its homotopy properties by means of its associated order complex $\mathcal{K}(X)$, whose simplices are the nonempty chains of $X$. If $x \in X$ and $Y \subseteq X$ is a subposet, let $Y_{\geq x}=\{y \in Y: y \geq x\}$. Define analogously $Y_{>x}, Y_{\leq x}$ and $Y_{<x}$. The link of $x$ in $Y$ is $Y_{<x} \cup Y_{>x}$.

Recall that if $f, g: X \rightarrow Y$ are two order-preserving maps between finite posets $X$ and $Y$ such that $f \leq g$ (i.e., $f(x) \leq g(x)$ for all $x \in X$ ), then $f$ and $g$ are homotopic when regarded as simplicial maps. Write $X \simeq Y$ if $\mathcal{K}(X) \simeq \mathcal{K}(Y)$. Note that this is not the usual convention that we used in our previous work $[11,13]$.

We recall Quillen's fibre lemma for finite posets. If $Y, X$ are sets, $X-Y$ denotes the complement of $Y$ in $X$.

Proposition 2.2 ([16, Proposition 1.6]). Let $f: X \rightarrow Y$ be an order-preserving map between finite posets. If $f^{-1}\left(Y_{\leq y}\right)$ is contractible for all $y \in Y$ (resp., $f^{-1}\left(Y_{\geq y}\right)$ is contractible for all $\left.y \in Y\right)$, then $f$ is a homotopy equivalence. In particular, if $X \subseteq X_{0}$ and $X_{>x}$ is contractible for all $x \in X_{0}-X$ (resp., $X_{<x}$ is contractible for all $\left.x \in X_{0}-X\right)$, then $X \hookrightarrow X_{0}$ is a homotopy equivalence.

The Brown poset $\mathcal{S}_{p}(G)$ is the poset of nontrivial $p$-subgroups of $G$. The inclusion $\mathcal{A}_{p}(G) \hookrightarrow \mathcal{S}_{p}(G)$ is a homotopy equivalence by [16, Proposition 2.1], and if $O_{p}(G) \neq 1$, then $\mathcal{A}_{p}(G)$ is contractible [16, Proposition 2.4].

Quillen related the direct product of groups with the join of their $p$-subgroup posets. The join of two posets $X * Y$ is the poset whose underlying set is the disjoint union of $X$ and $Y$, keeping the given ordering within $X$ and $Y$, and setting $x<y$ for each $x \in X$ and $y \in Y$. Moreover, $\mathcal{K}(X * Y)$ equals the join of simplicial complexes $\mathcal{K}(X) * \mathcal{K}(Y)$, and this is homeomorphic to the topological join of $\mathcal{K}(X)$ and $\mathcal{K}(Y)$ [16, Proposition 1.9]. If $Y \subseteq X$ are finite posets and $x \in X$, then note that the link of $x$ in $Y$ is the join $Y_{<x} * Y_{>x}$.

Proposition 2.3 ([16, Proposition 2.6]). $\mathcal{A}_{p}\left(G_{1} \times G_{2}\right) \simeq \mathcal{A}_{p}\left(G_{1}\right) * \mathcal{A}_{p}\left(G_{2}\right)$.

The following two results show that in some sense, it is enough to study the homotopical properties of $\mathcal{A}_{p}(G)$ when $Z(G)$ is the trivial group. Let $R=\mathbb{Z}$ or $\mathbb{Q}$.

Proposition 2.4. Let $Z \leq Z(G)$. The following hold:

1. If $Z$ is a nontrivial p-group, then $\mathcal{A}_{p}(G)$ is contractible.

2. If $Z$ is a $p^{\prime}$-group, then the induced map $\mathcal{A}_{p}(G) \rightarrow \mathcal{A}_{p}(G / Z)$ is an isomorphism of posets. Moreover, $O_{p}(G / Z) \cong O_{p}(G)$. 
3. In particular, if $G$ satisfies $(H 1)_{R}$ and $Z \neq 1$ is a $p^{\prime}$-group, then $G$ satisfies $(R-Q C)$, where $R=\mathbb{Z}$ or Q. Therefore, we may assume that $Z(G)=1$ under $(H 1)_{R}$ to study $(R-Q C)$.

Proof. Part 1 follows easily, since $O_{p}(Z) \leq O_{p}(G)$. Part 2 follows directly from the isomorphism theorems and Sylow's theorems. For the 'moreover' of part 2, note that if $H \leq G$, then $O_{p}(H Z / Z) \cong$ $O_{p}(H Z)$, since $Z$ is a central $p^{\prime}$-subgroup of $G$. Finally, part 3 is a consequence of the definition of the $(\mathrm{H} 1)_{R}$ hypothesis and part 2.

Next we give an immediate consequence of the $(\mathrm{H} 1)_{R}$ hypothesis.

Lemma 2.5. If $G$ satisfies $(H 1)_{R}$ and either $Z(G) \neq 1$ or $\Omega_{1}(G)<G$, then $G$ satisfies $(R-Q C)$.

Therefore, under $(H 1)_{R}$, we may assume that $Z(G)=1$ and $\Omega_{1}(G)=G$ to study $(R-Q C)$.

Proof. Suppose that $O_{p}(G)=1$. If $Z(G) \neq 1$, then $\mathcal{A}_{p}(G)=\mathcal{A}_{p}(G / Z(G))$ by the Proposition 2.4. Since $G / Z(G)$ is a proper central quotient of $G$, it satisfies $\left(R\right.$-QC) by $(\mathrm{H} 1)_{R}$. Moreover, we also have $O_{p}(G / Z(G))=O_{p}(G)=1$, so $\tilde{H}_{*}\left(\mathcal{A}_{p}(G), R\right)=\tilde{H}_{*}\left(\mathcal{A}_{p}(G / Z(G)), R\right) \neq 0$.

If $\Omega_{1}(G)<G$, then $\Omega_{1}(G)$ satisfies $\left(R\right.$-QC) by (H1) . Note that $O_{p}\left(\Omega_{1}(G)\right)=1$, since $\Omega_{1}(G)$ is normal in $G$. Since $\mathcal{A}_{p}(G)=\mathcal{A}_{p}\left(\Omega_{1}(G)\right), \tilde{H}_{*}\left(\mathcal{A}_{p}(G), R\right)=\tilde{H}_{*}\left(\mathcal{A}_{p}\left(\Omega_{1}(G)\right), R\right) \neq 0$.

In the lemmas to follow, we recall some results that will play a fundamental role in the proof of our main theorems. For a given subgroup $H \leq G$, we 'inflate' the subposet $\mathcal{A}_{p}(H)$ and then we show that the remaining points of $\mathcal{A}_{p}(G)$ are attached to this inflated subposet throughout their centralisers in $H$.

Definition 2.6. For $H \leq G$, define

$$
\mathcal{N}(H):=\left\{E \in \mathcal{A}_{p}(G): E \cap H \neq 1\right\} .
$$

We sometimes abbreviate $\mathcal{N}_{H}=\mathcal{N}(H)$.

We can also regard the poset $\mathcal{N}(H)$ as the 'neighbourhood' of $\mathcal{A}_{p}(H)$, and $\mathcal{N}(H)-\mathcal{A}_{p}(H)$ as the 'boundary' of this neighbourhood. We give some consequences of this definition, which were used for computing the examples given in [14] (compare [18, 19]).

Lemma 2.7. If $H \leq G$, then $\mathcal{A}_{p}(H) \hookrightarrow \mathcal{N}(H)$ is a strong deformation retract.

Proof. Let $i: \mathcal{A}_{p}(H) \hookrightarrow \mathcal{N}(H)$ be the inclusion and $\varphi: \mathcal{N}(H) \rightarrow \mathcal{A}_{p}(H)$ the map defined by $\varphi(E)=E \cap H$. Then $i$ and $\varphi$ are order-preserving maps with $i \varphi \leq \operatorname{Id}_{\mathcal{N}(H)}$ and $\varphi i=\operatorname{Id}_{\mathcal{A}_{p}(H)}$.

Next, we show that the elements outside $\mathcal{N}(H)$ attach to it via their centralisers in $H$.

Lemma 2.8. Let $H \leq G$ be a subgroup and let $E \in \mathcal{A}_{p}(G)$ be such that $E \cap H=1$. Then $\mathcal{N}(H)_{>E}$ is homotopy-equivalent to $\mathcal{A}_{p}\left(C_{H}(E)\right)$.

Proof. Let $f: \mathcal{A}_{p}\left(C_{H}(E)\right) \rightarrow \mathcal{N}(H)_{>E}$ and $g: \mathcal{N}(H)_{>E} \rightarrow \mathcal{A}_{p}\left(C_{H}(E)\right)$ be the maps defined by $f(A)=A E$ and $g(A)=A \cap H$. Then $f g(A)=(A \cap H) E \leq A$ and $g f(A)=(A E) \cap H=A$ (by modular law). Hence $f g \leq \operatorname{Id}_{\mathcal{N}(H)_{>E}}$ and $g f=\operatorname{Id}_{\mathcal{A}_{p}\left(C_{H}(E)\right)}$.

We can rebuild $\mathcal{A}_{p}(G)$ from $\mathcal{N}(H)$ by attaching points in the following way. Take a linear extension of the complement $\mathcal{A}_{p}(G)-\mathcal{N}(H)=\left\{E_{1}, \ldots, E_{r}\right\}$ such that $E_{i} \leq E_{j}$ implies $i \leq j$. For each $0 \leq i \leq r$, consider the subposet $X_{i}=\mathcal{N}(H) \cup\left\{E_{1}, \ldots, E_{i}\right\}$. This gives rise to a filtration

$$
\mathcal{N}(H)=X_{0} \subseteq X_{1} \subseteq \cdots \subseteq X_{r}=\mathcal{A}_{p}(G),
$$

where $X_{i}=X_{i-1} \cup\left\{E_{i}\right\}$ and the link of $E_{i}$ in $X_{i-1}$ is

$$
\left(\mathcal{A}_{p}\left(E_{i}\right)-\left\{E_{i}\right\}\right) * \mathcal{N}(H)_{>E_{i}} \simeq\left(\bigvee_{l=1}^{k_{i}} \mathbb{S}^{m_{p}\left(E_{i}\right)-2}\right) * \mathcal{A}_{p}\left(C_{H}\left(E_{i}\right)\right),
$$


with $k_{i}=p^{\left({ }^{m_{p}\left(E_{i}\right)}\right)}$. Recall that if $E$ is an elementary abelian $p$-group, $\mathcal{A}_{p}(E)-\{E\}$ is homotopyequivalent to a wedge of $\left.p^{\left(c_{p}(E)\right.}{ }_{2}\right)$ spheres of dimension $m_{p}(E)-2$ (compare [16, p. 58]).

This provides a useful way to study the homotopy type of $\mathcal{A}_{p}(G)$ if we select a convenient subgroup $H \leq G$ for which we understand the structure of these centralisers. In particular, if they are contractible, the homotopy type of $\mathcal{A}_{p}(H)$ does not change.

Lemma 2.9 (compare [14, Lemma 4.3]). Let $G$ be a finite group and let $H \leq G$. In addition, suppose that $O_{p}\left(C_{H}(E)\right) \neq 1$ for each $E \in \mathcal{A}_{p}(G)$ with $E \cap H=1$. Then $\mathcal{A}_{p}(G) \simeq \mathcal{A}_{p}(H)$.

Proof. Let $E \in \mathcal{A}_{p}(G)-\mathcal{N}(H)$. By Lemma 2.8, $\mathcal{N}(H)_{>E} \simeq \mathcal{A}_{p}\left(C_{H}(E)\right)$, which is contractible by hypothesis. Finally, by Proposition 2.2 and Lemma 2.7, $\mathcal{A}_{p}(G) \simeq \mathcal{N}(H) \simeq \mathcal{A}_{p}(H)$.

For example, we will usually take $H$ to be $L C_{G}(L)$, where $L$ is a simple component of $G$. Note that $F^{*}(G) \leq H$. If $E \in \mathcal{A}_{p}\left(N_{G}(L)\right)$ and $E \cap H=1$, then $C_{H}(E)=C_{L}(E) C_{G}(L E)$ and $\mathcal{A}_{p}\left(C_{H}(E)\right) \simeq$ $\mathcal{A}_{p}\left(C_{L}(E)\right) * \mathcal{A}_{p}\left(C_{G}(L E)\right)$. The group $C_{L}(E)$ is the centraliser of an elementary abelian $p$-group acting on the simple group $L$, which can be described by using the classification of the finite simple groups. We may also apply inductive arguments on $C_{G}(L E)$.

\section{The homology propagation lemma}

The aim of this section is to propose a generalisation of the homology propagation lemma [5, Lemma 0.27], stated in Lemma 3.14. Both lemmas allow us to propagate nonzero (free) homology from proper subposets to the whole Quillen poset. These tools will be very useful to establish Quillen's conjecture when we have an extra inductive assumption such as $(\mathrm{H} 1)_{R}$.

Our Lemma 3.14 shares the spirit of [5, Lemma 0.27] but with two extra features: it works for homology with coefficients in $\mathbb{Z}$, and it can be applied to suitable proper subposets $X \subset \mathcal{A}_{p}(G)$. This subposet $X$ will be typically chosen to be homotopy-equivalent to $\mathcal{A}_{p}(G)$ but better behaved, in a certain sense, than the Quillen poset. In many cases, we will see that $X$ satisfies the hypotheses of Lemma 3.14 while $\mathcal{A}_{p}(G)$ does not.

Before proceeding with the proof of this lemma, we need some definitions and generalisations of the results of [5]. From now on, we suppress the coefficient notation on the homology and suppose that they are taken in the ring $R=\mathbb{Z}$ or $\mathbb{Q}$. The definitions that follow do not depend on the coefficient ring.

Let $X$ be a finite poset. Recall that a chain of $X$ is a subset $a \subseteq X$ whose elements are pairwise comparable. We usually write $a=\left(x_{0}<x_{1}<\cdots<x_{n}\right)$ to emphasise the order of its elements. Denote by max $a$ and $\min a$ the maximum and minimum element of $a$, respectively, if $a$ is nonempty. Let $X^{\prime}$ be the poset of nonempty chains of $X$. Equivalently, $X^{\prime}$ is the face poset of $\mathcal{K}(X)$.

Denote by $\tilde{C}_{*}(X)$ the augmented chain complex of $X$ with coefficients in $R$. Recall that $\tilde{C}_{n}(X)$ is freely generated by the chains $\left(x_{0}<x_{1}<\cdots<x_{n}\right)$ in $X$. Write $\tilde{Z}_{n}(X)$ for the subgroup of $n$-cycles and $\tilde{H}_{*}(X)$ for the reduced homology of $X$. We say that a chain $a \in X^{\prime}$ is an addend of $\alpha \in \tilde{C}_{n}(X)$, and we write $a \in \alpha$, if $a$ appears with nonzero coefficient in the sum decomposition of $\alpha$ in the canonical basis of $\tilde{C}_{n}(X)$.

Definition 3.1. Let $X$ be a finite poset. A chain $a \in X^{\prime}$ is full if for every $x \in X$ such that $\{x\} \cup a$ is a chain, we have $x \in a$ or $x \geq \max a$. A chain $b$ containing $a$ is called an $a$-initial chain if for every $x \in b-a$, we have $x>\max a$.

The following property was introduced by Aschbacher and Smith in [5].

Definition 3.2. We say that $G$ satisfies the Quillen dimension property at $p,(\mathcal{D D})_{p}$ for short, if $\tilde{H}_{m_{p}(G)-1}\left(\mathcal{A}_{p}(G)\right) \neq 0$. That is, $\mathcal{A}_{p}(G)$ has nonzero homology in the highest possible degree.

Observe that the top integer homology group of $\mathcal{A}_{p}(G)$ is always free, so this definition does not depend on the chosen coefficient ring $\mathbb{Z}$ or $\mathbb{Q}$. It is worth noting that finite groups may not satisfy $(Q \mathcal{D})_{p}$ in general; this was already observed by Quillen in [16]. 
Definition 3.3. Suppose that $G$ satisfies $(Q \mathcal{D})_{p}$ and let $m=m_{p}(G)-1$. If $\alpha \in \tilde{H}_{m}\left(\mathcal{A}_{p}(G)\right)=$ $\tilde{Z}_{m}\left(\mathcal{A}_{p}(G)\right)$ is a nonzero cycle and $a \in \alpha$ is an addend of $\alpha$, we say that $a$ or max $a$ exhibits $(Q \mathcal{D})_{p}$ for $G$. Note that $a$ is a full chain.

Next, we recall a special configuration of the $p$-solvable case of the conjecture. Its proof depends on the classification of the finite simple groups and can be found in [20, Theorem 8.2.12] (see also [1, 5, 7]).

Theorem 3.4. If $G=O_{p^{\prime}}(G) A$, where $A$ is an elementary abelian p-group acting faithfully on $O_{p^{\prime}}(G)$, then $G$ satisfies $(Q \mathcal{D})_{p}$ exhibited by $A$.

Now we set up the proper context that we need to culminate in the proof of Lemma 3.14. We shall work under [5, Hypothesis 0.15], which we state below as Hypothesis 3.5.

Hypothesis 3.5 (Central product). $H \leq G$ and $K \leq C_{G}(H)$, with $H \cap K$ a $p^{\prime}$-group.

Hypothesis 3.5 implies that $[H, K]=1$ and $H \cap K \leq Z(H) \cap Z(K)$. Moreover, we have

$$
\mathcal{A}_{p}(H K) \simeq \mathcal{A}_{p}(H / H \cap K) * \mathcal{A}_{p}(K / H \cap K),
$$

since $H K$ is a central product and the shared central subgroup $H \cap K$ is a $p^{\prime}$-group [5, Lemma 0.11].

Under appropriate circumstances, there will be nonzero cycles $\alpha$ and $\beta$ in the homology of $\mathcal{A}_{p}(H)$ and $\mathcal{A}_{p}(K)$, respectively, and they will give rise to a nonzero cycle $\alpha \times \beta$ (the shuffle product) in the homology of $\mathcal{A}_{p}(H K)$. The final goal is to show that this product cycle produces nonzero homology for $\mathcal{A}_{p}(G)$. In order to do that, we will ask for some subgroup $A \in \mathcal{A}_{p}(H)$ involved in $\alpha$ to satisfy suitable strong hypotheses. With these hypotheses, if $\alpha \times \beta$ is the zero cycle (i.e., a boundary) in $\tilde{H}_{*}\left(\mathcal{A}_{p}(G)\right)$, we will reduce to a calculation in $\tilde{H}_{*}\left(\mathcal{A}_{p}(K)\right)$ and then arrive at a contradiction.

The idea of this section is to perform these homology computations in a typically proper subposet $X$, which in general will be constructed to be homotopy-equivalent to $\mathcal{A}_{p}(G)$. Hence, showing that $\alpha \times \beta$ is a nonzero cycle in $\tilde{H}_{*}(X)$ will lead to nonzero homology in $\mathcal{A}_{p}(G)$, as desired.

The reduction described is in fact carried out inside the subposet $\mathcal{N}(K)$, lying over $\mathcal{A}_{p}(K)$. We shall take $X$ containing $\mathcal{N}(K)$.

Definition 3.6. Under Hypothesis 3.5, we call a subposet $X \subseteq \mathcal{A}_{p}(G)$ an $\mathcal{N}_{K}$-superset if $\mathcal{N}(K) \subseteq X$.

We proceed now to generalise the definitions and results coming after [5, Hypothesis 0.15].

Definition 3.7 ([5, Definition 0.19]). Assume Hypothesis 3.5. Let $a=\left(A_{0}<\cdots<A_{m}\right)$ and $b=\left(B_{0}<\right.$ $\left.\cdots<B_{n}\right)$ be chains of $\mathcal{A}_{p}(H)$ and $\mathcal{A}_{p}(K)$, respectively. We define the following chain in $\mathcal{A}_{p}(H K)$ :

$$
a * b:=\left(A_{0}<\cdots<A_{m}<B_{0} A_{m}<\cdots<B_{n} A_{m}\right) .
$$

Let $c=(0,1,2, \ldots, m+n+1)$. A shuffle is a permutation $\sigma$ of the set $\{0,1,2, \ldots, m+n+1\}$ such that $\sigma(i)<\sigma(j)$ if $i<j \leq m$ or $m+1 \leq i<j$. Let $\sigma(c):=(\sigma(0), \sigma(1), \ldots, \sigma(m+n+1))$.

With the notation of the Definition 3.7, let $C_{j}=A_{j}$ if $j \leq m$ or $B_{j-(m+1)}$ if $j \geq m+1$. For a shuffle $\sigma$, define $(a \times b)_{\sigma}$ to be the chain whose $i$ th element is $C_{\sigma(0)} C_{\sigma(1)} \cdots C_{\sigma(i)}$.

Definition 3.8 ([5, Definition 0.21]). Assume Hypothesis 3.5. The shuffle product of $a$ and $b$ is

$$
a \times b:=\sum_{\sigma \text { shuffle }}(-1)^{\sigma}(a \times b)_{\sigma} \in \tilde{C}_{m+n+1}\left(\mathcal{A}_{p}(H K)\right) .
$$

Extend this definition by linearity to the tensor product of the chain complexes $\tilde{C}_{*}\left(\mathcal{A}_{p}(H)\right)$ and $\tilde{C}_{*}\left(\mathcal{A}_{p}(K)\right)$.

Recall that we are aiming to apply Lemma 3.14 which, in contrast to [5, Lemma 0.27], works with a potentially proper $\mathcal{N}_{K}$-superset $X$ of $\mathcal{A}_{p}(G)$. In many situation it will be the case that $\mathcal{A}_{p}(H) \subseteq X$, 
hence $\tilde{C}_{*}\left(\mathcal{A}_{p}(H)\right) \subseteq \tilde{C}_{*}(X)$ and the following lemmas are automatic. However, our overall arguments do not require this assumption, so we supply the lemmas to also cover these cases.

Lemma 3.9. Assume Hypothesis 3.5 and let $X$ be an $\mathcal{N}_{K}$-superset.

(i) If $a \in X^{\prime} \cap \mathcal{A}_{p}(H)^{\prime}, b \in \mathcal{A}_{p}(K)^{\prime}$ and $\sigma$ is a shuffle, then $(a \times b)_{\sigma} \in X^{\prime}$.

(ii) If $\alpha \in \tilde{C}_{*}(X) \cap \tilde{C}_{*}\left(\mathcal{A}_{p}(H)\right)$ and $\beta \in \tilde{C}_{*}\left(\mathcal{A}_{p}(K)\right)$, then $\alpha \times \beta \in \tilde{C}_{*}(X) \cap \tilde{C}_{*}\left(\mathcal{A}_{p}(H K)\right)$.

Proof. If $C \in(a \times b)_{\sigma}$, then either $C \in a \subseteq X$ or else $C$ contains some subgroup $B \in b$. In the latter case, $C \cap K \geq B \neq 1$, so $C \in \mathcal{N}(K) \subseteq X$, since $X$ is an $\mathcal{N}_{K}$-superset. This proves part (i). Part (ii) follows from (i), by $\tilde{C}_{*}(X) \cap \tilde{C}_{*}\left(\mathcal{A}_{p}(H)\right)=\tilde{C}_{*}\left(X \cap \mathcal{A}_{p}(H)\right)$ and a linearity argument.

Proposition 3.10 (compare [5, Corollary 0.23]). Under Hypothesis 3.5, if $\alpha \in \tilde{Z}_{m}\left(\mathcal{A}_{p}(H)\right)$ and $\beta \in \tilde{Z}_{n}\left(\mathcal{A}_{p}(K)\right)$, then $\alpha \times \beta \in \tilde{Z}_{m+n+1}\left(\mathcal{A}_{p}(H K)\right)$. In addition, if $X$ is an $\mathcal{N}_{K}$-superset and $\alpha \in \tilde{C}_{*}(X)$, then $\alpha \times \beta \in \tilde{Z}_{m+n+1}(X)$.

Proof. The first part is [5, Corollary 0.23], and the second part follows from Lemma 3.9.

Remark 3.11. Let $X$ be a finite poset and let $a \in X^{\prime}$. Denote by $\tilde{C}_{*}(X)_{a}$ the subgroup of $a$-initial chains and by $\tilde{C}_{*}(X)_{\neg a}$ the subgroup of non- $a$-initial chains. Clearly we have a decomposition

$$
\tilde{C}_{*}(X)=\tilde{C}_{*}(X)_{a} \oplus \tilde{C}_{*}(X)_{\neg a} .
$$

Moreover, if $\partial$ denotes the boundary map of its chain complex, then

$$
\partial\left(\tilde{C}_{*}(X)_{\neg a}\right) \subseteq \tilde{C}_{*}(X)_{\neg a} .
$$

If $\gamma \in \tilde{C}_{*}(X)$, then $\gamma=\gamma_{a}+\gamma_{\neg a}$, where $\gamma_{a}$ corresponds to the $a$-initial part of $\gamma$, and

$$
\partial \gamma=\partial\left(\gamma_{a}\right)+\partial\left(\gamma_{\neg a}\right)=\left(\partial\left(\gamma_{a}\right)\right)_{a}+\left(\partial\left(\gamma_{a}\right)\right)_{\neg a}+\partial\left(\gamma_{\neg a}\right) .
$$

This remark yields the following lemma.

Lemma 3.12 (compare [5, Lemma 0.24]). If $a \in X^{\prime}$ is a full chain, then $(\partial \gamma)_{a}=\left(\partial \gamma_{a}\right)_{a}$.

The following lemma generalises [5, Lemma 0.25(i)] to $\mathcal{N}_{K}$-supersets.

Lemma 3.13 (compare [5, Lemma 0.25]). Assume Hypothesis 3.5.

(i) If $a \in \mathcal{A}_{p}(H)^{\prime}$ and $b \in \mathcal{A}_{p}(K)^{\prime}$, then $(a \times b)_{a}=(a \times b)_{\sigma=i d}=a * b$ in $\tilde{C}_{*}\left(\mathcal{A}_{p}(H K)\right) \subseteq \tilde{C}_{*}\left(\mathcal{A}_{p}(G)\right)$.

(ii) In addition, if $X$ is an $\mathcal{N}_{K}$-superset and $a \in X^{\prime}$, then the conclusion of $(i)$ remains true in $\tilde{C}_{*}(X)$.

Proof. Let $\sigma$ be a shuffle and let $C \in(a \times b)_{\sigma}$ with $C \notin a$. Then $C \geq B$ for some $B \in b$, so $C \in \mathcal{N}(K)$ (see the proof of Lemma 3.9). Since $H \cap K$ is a $p^{\prime}$-group, $C \notin \mathcal{A}_{p}(H)$. Therefore, $(a \times b)_{\sigma}$ is $a$-initial if and only if $\sigma=\mathrm{id}$, proving part (i). Part (ii) follows from Lemma 3.9.

We prove now the mentioned generalisation of the fundamental homology propagation lemma [5, Lemma 0.27]. Recall that we are working with coefficients in $R=\mathbb{Z}$ or $\mathbb{Q}$.

Lemma 3.14. Let $G$ be a finite group. Let $H, K \leq G$ and $X \subseteq \mathcal{A}_{p}(G)$ be such that

(i) $H$ and $K$ satisfy Hypothesis 3.5;

(ii) $X$ is an $\mathcal{N}_{K}$-superset;

(iii) there exist a chain $a \in \mathcal{A}_{p}(H)^{\prime} \cap X^{\prime}$ and a cycle $\alpha \in \tilde{C}_{m}\left(\mathcal{A}_{p}(H)\right) \cap \tilde{C}_{m}(X)$ such that the coefficient of a in $\alpha$ is invertible and $\alpha \neq 0$ in $\tilde{H}_{m}\left(\mathcal{A}_{p}(H)\right)$ (for some $m \geq-1$ );

(iv) such $a$ is a full chain in $X$ and $X_{>\max a} \subseteq \mathcal{N}(K)$; and

(v) $\tilde{H}_{*}\left(\mathcal{A}_{p}(K)\right) \neq 0$.

Then $\tilde{H}_{*}(X) \neq 0$. 
In particular, under (i), hypotheses (ii)-(iv) hold if

(a) coefficients are taken in $\mathbb{Q}$,

(b) $X=\mathcal{A}_{p}(G)$ and

(c) $H$ has $(Q \mathcal{D})_{p}$ exhibited by $A \in \mathcal{A}_{p}(H)$ such that $\mathcal{A}_{p}(G)_{>A} \subseteq A \times K$.

(This is the hypothesis in [5, Lemma 0.27], so the present result is indeed a generalisation.)

Proof. We essentially carry out the original proof of [5, Lemma 0.27$]$ inside $\tilde{C}_{*}(X)$, since $X$ is an $\mathcal{N}_{K}$-superset.

By hypothesis $(\mathrm{v})$, there exists a cycle $\beta \in \tilde{C}_{n}\left(\mathcal{A}_{p}(K)\right)$ which is not a boundary in $\tilde{C}_{*}\left(\mathcal{A}_{p}(K)\right)$. Choose a chain $a$ and a cycle $\alpha$ as in hypothesis (iii). Then $\alpha \times \beta \in \tilde{Z}_{m+n+1}(X)$, by hypotheses (i)-(iii) and Proposition 3.10. We show that $\alpha \times \beta$ gives a nonzero cycle in the homology of $X$.

Suppose by way of contradiction that for some chain $\gamma \in \tilde{C}_{m+n+2}(X)$ we have

$$
\alpha \times \beta=\partial \gamma
$$

Write $\beta=\sum_{i} q_{i}\left(B_{0}^{i}<\cdots<B_{n}^{i}\right)$ and $\gamma=\sum_{j \in J} p_{j}\left(C_{0}^{j}<\cdots<C_{m+n+2}^{j}\right)$. Now take $a$-initial parts in both sides of equation (3.1):

$$
(\alpha \times \beta)_{a}=(\partial \gamma)_{a}
$$

Note that no intermediate group lying in $X$ can be added within $a$, due to hypothesis (iv). Let $A=\max a$. By Lemma 3.13(ii), the left-hand side of equation (3.2) becomes

$$
(\alpha \times \beta)_{a}=q(a \times \beta)=q \sum_{i} q_{i} a \cup\left(A B_{0}^{i}<\cdots<A B_{n}^{i}\right),
$$

where by hypothesis (iii), $q \neq 0$ is the coefficient of $a$ in $\alpha$, and it is invertible. Equation (3.3) is then equal to the right-hand side of equation (3.2), which using Lemma 3.12 is

$$
\begin{aligned}
(\partial \gamma)_{a} & =\sum_{j \in J^{\prime}} p_{j} \sum_{k=m+1}^{m+n+2}(-1)^{k} a \cup\left(C_{m+1}^{j}<\cdots<\hat{C}_{k}^{j}<\cdots<C_{m+n+2}^{j}\right) \\
& =\sum_{j \in J^{\prime}} p_{j}(-1)^{m+1} \sum_{k=0}^{n+1}(-1)^{k} a \cup\left(C_{m+1}^{j}<\cdots<\hat{C}_{k+m+1}^{j}<\cdots<C_{m+n+2}^{j}\right) .
\end{aligned}
$$

The hat notation $\hat{C}_{k}^{j}$ means that this term does not appear in the chain, and $J^{\prime}=$ $\left\{j \in J: a \subseteq\left(C_{0}^{j}<\cdots<C_{m+n+2}^{j}\right)\right\}$. For $0 \leq k \leq n+1$, set $D_{k}^{j}:=C_{k+m+1}^{j}$. Since $C_{k+m+1}^{j}>A$, we have $D_{k}^{j} \in \mathcal{N}(K)$ by hypothesis (iv), so that $D_{k}^{j} \cap K \neq 1$.

We use now the $\sim$ operation, which maps an $a$-initial chain to its subchain beginning just after $\max a$. Apply the $\sim$ operation on both sides of the equation of $a$-initial chains (3.2) using equations (3.3) and (3.4), respectively. The left-hand side of equation (3.2) becomes $q \tilde{\beta}$, where

$$
\tilde{\beta}=\sum_{i} q_{i}\left(A B_{0}^{i}<\cdots<A B_{n}^{i}\right) \in \tilde{C}_{*}\left(\mathcal{A}_{p}(\mathcal{N}(K))\right) .
$$

The right-hand side of equation (3.2) becomes $\partial \tilde{\gamma}$, with

$$
\tilde{\gamma}=\sum_{j \in J^{\prime}} p_{j}(-1)^{m+1}\left(D_{0}^{j}<\cdots<D_{n+1}^{j}\right) \in \tilde{C}_{*}\left(\mathcal{A}_{p}(\mathcal{N}(K))\right) .
$$


Now we reduce the homology computation in $\mathcal{N}(K)$ to a calculation in $\tilde{H}_{*}\left(\mathcal{A}_{p}(K)\right)$. Consider the homotopy equivalence given by the poset map $\varphi: \mathcal{N}(K) \rightarrow \mathcal{A}_{p}(K)$ of Lemma 2.7. Denoting by $\varphi_{*}$ the induced map in the chain complexes, we get the following equalities in $\tilde{C}_{*}\left(\mathcal{A}_{p}(K)\right)$ :

$$
q \beta=\varphi_{*}(q \tilde{\beta})=\varphi_{*}(\partial(\tilde{\gamma}))=\partial\left(\varphi_{*}(\tilde{\gamma})\right),
$$

where $\varphi_{*}(\tilde{\gamma}) \in \tilde{C}_{n+1}\left(\mathcal{A}_{p}(K)\right)$. Since $q$ is invertible, we have found that $\beta$ is a boundary in the chain complex $\tilde{C}_{*}\left(\mathcal{A}_{p}(K)\right)$, contradicting our initial assumption on $\beta$.

Note that Lemma 3.14 does not require $H$ to have $(Q \mathcal{D})_{p}$, which by contrast was fundamental in [5, Lemma 0.27]. This assumption is relaxed in hypotheses (iii) and (iv).

Remark 3.15. If coefficients are taken in $\mathbb{Q}$, then the coefficient requirement in hypothesis (iii) is automatically guaranteed if $a \in \alpha$. If they are taken in $\mathbb{Z}$, then hypothesis (iii) implies that the coefficient of $a \in \alpha$ is \pm 1 . We may eliminate this restriction if we can take $\beta \in \tilde{H}_{*}\left(\mathcal{A}_{p}(K), \mathbb{Z}\right)$ of order prime to the coefficient $q$ of $a$ in $\alpha$ by equation (3.5) (or if it is not a torsion element).

Remark 3.16. Indeed, getting Lemma 3.14(v) is in general the hard part. In [5], this hypothesis is frequently obtained by applying [5, Theorem 2.4], which has certain restrictions on the prime $p$. One of our goals is to try to avoid restrictions on $p$, so we investigate other methods to get this hypothesis.

\section{The reduction $O_{p^{\prime}}(G)=1$}

In this section we show that if $G$ satisfies $(\mathrm{H} 1)_{R}$ and $O_{p^{\prime}}(G) \neq 1$, then $G$ satisfies $(R$-QC), with $R=\mathbb{Z}$ or $\mathbb{Q}$. This reduces the study of $(R-\mathrm{QC})$ to finite groups $G$ with $O_{p^{\prime}}(G)=1$.

This is motivated by the original result [5, Proposition 1.6], stated for $p>5$ and $R=\mathbb{Q}$. We prove a more general version of this fact by using Lemma 3.14, without those restrictions on the prime $p$ and for $R=\mathbb{Z}$ or $\mathbb{Q}$. In the proof, we will construct a subposet $X$ of $\mathcal{A}_{p}(G)$ satisfying the hypotheses of Lemma 3.14. This route is comparatively elementary in contrast with [5, Proposition 1.6], which quotes the strongly CFSG-dependent result [5, Theorem 2.4]. Our proof does not depend on that result, and we will only need to quote Theorem 3.4, which uses only easy facts about coprime automorphisms of the simple groups (whereas [5, Theorem 2.4] requires much deeper details about the structure of the simple groups).

Theorem 4.1. Suppose that $G$ satisfies $(H 1)_{R}$ and that $O_{p^{\prime}}(G) \neq 1$. Then $G$ satisfies $(R-Q C)$.

Proof. Suppose that $G$ satisfies $(\mathrm{H} 1)_{R}$ and that $O_{p^{\prime}}(G) \neq 1$. Assume that $O_{p}(G)=1$. Our goal is to show that $\tilde{H}_{*}\left(\mathcal{A}_{p}(G), R\right) \neq 0$, with coefficients in $R=\mathbb{Z}$ or $\mathbb{Q}$.

First note that if $H<G$ is a proper normal subgroup such that $\mathcal{A}_{p}(H) \simeq \mathcal{A}_{p}(G)$, then $O_{p}(H) \leq$ $O_{p}(G)=1$ and hence, by $(\mathrm{H} 1)_{R}, 0 \neq \tilde{H}_{*}\left(\mathcal{A}_{p}(H), R\right) \cong \tilde{H}_{*}\left(\mathcal{A}_{p}(G), R\right)$. Therefore, we can further assume that no such subgroup exists:

(H2) If $H<G$ is a proper normal subgroup, then $\mathcal{A}_{p}(H) \nsucc \mathcal{A}_{p}(G)$.

Now we head to the construction of a homotopy-equivalent subposet $X$ of $\mathcal{A}_{p}(G)$ to apply Lemma 3.14 and get our goal. To achieve this, we are going to deduce a series of properties on our group $G$ which will lead to the choice of convenient subgroups $H$ and $K$ and the definition of $X$ satisfying the hypotheses of Lemma 3.14. In view of Lemma 2.5, we can suppose that

$$
Z(G)=1 \text { and } \Omega_{1}(G)=G .
$$

Let $L:=O_{p^{\prime}}(G)$, which is nontrivial by hypothesis. The following claim holds by (H3).

Claim 1. $C_{G}(L)<G$, and hence some $A \in \mathcal{A}_{p}(G)$ acts faithfully on $L$. 
Recall that for $A \in \mathcal{A}_{p}(G)$, we have $O_{p}(L A)=C_{A}(L)$, since $A$ is a Sylow $p$-subgroup of $L A$. Moreover, $A$ acts faithfully on $L$ if and only if $C_{A}(L)=1$. Let

$$
\begin{gathered}
\mathcal{F}=\left\{A \in \mathcal{A}_{p}(G): A \text { acts faithfully on } L\right\}, \\
\mathcal{N}=\left\{A \in \mathcal{A}_{p}(G): A \text { acts nonfaithfully on } L\right\} .
\end{gathered}
$$

The following claim is immediate from these definitions.

Claim 2. $\mathcal{F}$ and $\mathcal{N}$ are disjoint, $\mathcal{A}_{p}(G)=\mathcal{F} \cup \mathcal{N}, \mathcal{F}$ is nonempty by Claim 1 and $\mathcal{N}=\mathcal{N}\left(C_{G}(L)\right)$.

With an eye on the notation of Lemma 3.14, for $A \in \mathcal{F}$ define $H_{A}:=L A$ and $K_{A}:=C_{G}\left(H_{A}\right)$. The following claim gives some properties of $H_{A}$ and $K_{A}$.

Claim 3. $H_{A}$ and $K_{A}$ satisfy Hypothesis $3.5, K_{A}=C_{C_{G}(L)}(A)$ and $\mathcal{N}_{>A}=\mathcal{N}\left(K_{A}\right)_{>A}$.

Proof. Note that $C_{G}(L A)=C_{C_{G}(L)}(A)$ and that $H_{A} \cap K_{A} \leq Z\left(H_{A}\right)=Z(L A)$ is a $p^{\prime}$-group since $O_{p}(L A)=C_{A}(L)=1$. Therefore $H_{A}$ and $K_{A}$ satisfy Hypothesis 3.5.

Since $C_{G}(L A) \leq C_{G}(L)$, we have $\mathcal{N}\left(K_{A}\right) \subseteq \mathcal{N}\left(C_{G}(L)\right)=\mathcal{N}$. Hence $\mathcal{N}\left(K_{A}\right)_{>A} \subseteq \mathcal{N}>_{A}$. Conversely, if $B \in \mathcal{N}_{>A}$, then $B \leq C_{G}(A)$ and $C_{B}(L) \neq 1$. Hence, $1 \neq C_{B}(L)=C_{G}(A) \cap B \cap C_{G}(L)=$ $B \cap C_{G}(L A)=B \cap K_{A}$.

Now we will see how the configuration of Lemma 3.14 brings new ideas beyond the analogous result of [5]. We show next how to get its hypothesis (v) (see Remark 3.16).

Claim 4. There is $A \in \mathcal{F}$ with $O_{p}\left(K_{A}\right)=1$.

Proof. If for all $A \in \mathcal{F}$ we have $O_{p}\left(C_{C_{G}(L)}(A)\right)=O_{p}\left(K_{A}\right) \neq 1$, then by Claim 2 and Lemma 2.9, we get $\mathcal{A}_{p}(G) \simeq \mathcal{A}_{p}\left(C_{G}(L)\right)$. This contradicts $(\mathrm{H} 2)$, since $C_{G}(L)$ is normal in $G$.

Next we define the subposet $X$ by removing points of $\mathcal{A}_{p}(G)$ with contractible link, so we preserve the homotopy type. By Claim 4 , we can take $A \in \mathcal{F}$ of maximal $p$-rank subject to $O_{p}\left(K_{A}\right)=1$. Let $X=\mathcal{A}_{p}(G)-\mathcal{F}_{>A}=\mathcal{N} \cup\left(\mathcal{F}-\mathcal{F}_{>A}\right)$. Note that $\mathcal{N} \cap\left(\mathcal{F}-\mathcal{F}_{>A}\right)=\emptyset$, by Claim 2.

Claim 5. If $B \in \mathcal{A}_{p}(G)-X$, then $X_{>B}=\mathcal{N}_{>B}$ is contractible. In particular, $X \simeq \mathcal{A}_{p}(G)$.

Proof. If $B \in \mathcal{A}_{p}(G)-X=\mathcal{F}_{>A}$, then by the consequences already noted, $X_{>B}=\mathcal{N}_{>B}$, since $\mathcal{F}_{>B} \subseteq \mathcal{F}_{>A}$ and $\mathcal{A}_{p}(G)_{>B}=\mathcal{F}_{>B} \cup \mathcal{N}_{>B}$. It follows from Lemma 2.8 that

$$
X_{>B}=\mathcal{N}_{>B} \simeq \mathcal{A}_{p}\left(C_{C_{G}(L)}(B)\right)=\mathcal{A}_{p}\left(K_{B}\right) \simeq *,
$$

since $O_{p}\left(K_{B}\right) \neq 1$. Finally, by Proposition 2.2, $X \simeq \mathcal{A}_{p}(G)$.

Claim 6. $\mathcal{A}_{p}(L A) \subseteq X$.

Proof. Set $B \in \mathcal{A}_{p}(L A)$. Since $A$ is a Sylow $p$-subgroup of $L A$, there exists $g \in L$ such that $B \leq A^{g}$, so $C_{B}(L) \leq C_{A^{g}}(L)=\left(C_{A}(L)\right)^{g}=1$. Therefore $B$ is faithful on $L-$ that is, $B \in \mathcal{F}$, and $|B| \leq|A|$. Hence $B \notin \mathcal{F}_{>A}$, which means that $B \in X$.

We check the hypotheses of Lemma 3.14 with $H=H_{A}=L A, K=K_{A}=C_{G}(L A)$ and the subposet $X$.

(i) It holds by Claim 2 .

(ii) If $B \notin X$, then $B$ acts faithfully on $L$, so $1=C_{B}(L) \geq C_{B}(L A)=B \cap K$. In consequence, $\mathcal{N}(K) \subseteq X$ and $X$ is an $\mathcal{N}_{K}$-superset. 
(iii) By Theorem 3.4 applied to $H=L A$, we can pick a nonzero element $\alpha \in \tilde{H}_{m}\left(\mathcal{A}_{p}(H)\right.$, $\left.R\right)$, where $m=m_{p}(A)-1$. Since $\tilde{Z}_{m}\left(\mathcal{A}_{p}(H)\right)=\tilde{H}_{m}\left(\mathcal{A}_{p}(H), R\right), \alpha$ is actually a cycle, and by a dimension argument it involves a full chain $a$. Since $A$ is a Sylow $p$-subgroup of $H$, after conjugating $\alpha$ we may suppose that $A \in a$. Moreover, by Claim $6, \tilde{C}_{*}\left(\mathcal{A}_{p}(H)\right) \subseteq \tilde{C}_{*}(X)$. The coefficient of $a$ in $\alpha$ is invertible if $R=\mathbb{Q}$. For $R=\mathbb{Z}$ this is also true, but it is less immediate and depends on the results of [7] (see later for further details).

(iv) By (iii), $a$ is a full chain, $A=\max a$ and $X_{>A}=\mathcal{N}\left(K_{A}\right)_{>A}$ by Claims 2 and 3 and the consequences already noted.

(v) It holds by $(\mathrm{H} 1)_{R}$, since $O_{p}\left(K_{A}\right)=1$ by the choice of $A$ satisfying Claim 4 , and $K_{A}=C_{G}(L A) \leq$ $C_{G}(L)<G$ by Claim 1 .

By Claim 5 and Lemma 3.14, $\tilde{H}_{*}\left(\mathcal{A}_{p}(G), R\right) \cong \tilde{H}_{*}(X, R) \neq 0$.

We explain now how to obtain the invertible coefficient for $a$ in $\alpha$ if $R=\mathbb{Z}$, in order to fulfil hypothesis (iii) of Lemma 3.14. In the proof of (iii), we begin by fixing the cycle $\alpha$ and then we choose a chain $a \in \alpha$, which is always a full chain since $\alpha$ lies in the top degree chain group. Hence we need to show that for some $a \in \alpha$, its coefficient is equal to \pm 1 (see Remark 3.15). This is possible by using the explicit description of a nontrivial cycle that Díaz Ramos gave for the $p$-solvable case in [7]. It follows from the proofs of [7, Theorems 5.1, $5.3 \& 6.6]$.

This concludes the proof of the theorem for both versions of the conjecture.

We give some applications of Theorem 4.1. The following results depend on the results obtained on the fundamental group of the $p$-subgroup posets [12] and the almost-simple case of the conjecture [4].

Theorem 4.2 (compare [12, Theorem 5.2]). If $G$ is not an almost-simple group and $O_{p^{\prime}}(G)=1$, then $\pi_{1}\left(\mathcal{A}_{p}(G)\right)$ is a free group.

Theorem 4.3 ([4]). If $G$ is an almost-simple group, then $\tilde{H}_{*}\left(\mathcal{A}_{p}(G), \mathbb{Q}\right) \neq 0$.

Corollary 4.4. Suppose that $G$ satisfies $(H 1)_{R}$, with $R=\mathbb{Z}$ or $\mathbb{Q}$, and that $\mathcal{A}_{p}(G)$ is not simply connected. Then $G$ satisfies $(R-Q C)$.

Proof. Suppose that $O_{p}(G)=1$. The result clearly holds if $\mathcal{A}_{p}(G)$ is not connected, since $\tilde{H}_{0}\left(\mathcal{A}_{p}(G), \mathbb{Z}\right)$ is a nonzero free group in that case. If $O_{p^{\prime}}(G) \neq 1$, then $G$ satisfies $(R-\mathrm{QC})$ by Theorem 4.1. On the other hand, if $G$ is almost simple, then we are done by Theorem 4.3.

Therefore we can assume that $\mathcal{A}_{p}(G)$ is connected, $O_{p^{\prime}}(G)=1$ and $G$ is not almost simple. By Theorem 4.2, $\pi_{1}\left(\mathcal{A}_{p}(G)\right)$ is a free group, and since $\mathcal{A}_{p}(G)$ is connected but not simply connected, we also have $\pi_{1}\left(\mathcal{A}_{p}(G)\right) \neq 1$. Finally, by the Hurewicz isomorphism in the first homology group, we conclude that $\tilde{H}_{1}\left(\mathcal{A}_{p}(G), \mathbb{Z}\right)$ is a nonzero free group. Hence, $G$ satisfies $(R$-QC).

\section{Now we prove Theorem 2.}

Proof of Theorem 2. We only need to prove that if the original conjecture (QC) holds for all finite groups, then the integer homology version $(\mathbb{Z}-\mathrm{QC})$ holds.

Let $G$ be a group with $O_{p}(G)=1$. We shall prove that $\tilde{H}_{*}\left(\mathcal{A}_{p}(G), \mathbb{Z}\right) \neq 0$. By induction, we can assume that $(\mathbb{Z}-\mathrm{QC})$ holds for every group $H$ with $|H|<|G|$, so $G$ satisfies $(\mathrm{H} 1)_{\mathbb{Z}}$. By Corollary 4.4 we can also suppose that $\mathcal{A}_{p}(G)$ is simply connected. Since $G$ satisfies (QC), some of its homotopy groups are nontrivial, so by the Hurewicz theorem $\tilde{H}_{*}\left(\mathcal{A}_{p}(G), \mathbb{Z}\right) \neq 0$.

We close this section by using the rational version of Theorem 4.1 to extend some results of [14] on the integer conjecture $(\mathbb{Z}-\mathrm{QC})$ to the rational conjecture $(\mathbb{Q}-\mathrm{QC})$. We recall some of the main results of [14]. 
Theorem 4.5 ([14, Corollary 3.3]). Suppose that $\mathcal{K}\left(\mathcal{S}_{p}(G)\right)$ is homotopy-equivalent to a 2-dimensional and $G$-invariant subcomplex. Then $G$ satisfies $(\mathbb{Z}-Q C)$.

Corollary 4.6 ([14, Corollary 3.4]). The integer Quillen conjecture (ZZ-QC) holds for groups of p-rank at most 3 .

Corollary 4.7. Suppose that $G$ satisfies $(H 1)_{\mathbb{Q}}$ and that $\mathcal{K}\left(\mathcal{S}_{p}(G)\right)$ is homotopy-equivalent to a 2 dimensional and $G$-invariant subcomplex. Then $G$ satisfies $(\mathbb{Q}-Q C)$.

Proof. Suppose that $O_{p}(G)=1$. We show that $\mathcal{A}_{p}(G)$ is not $\mathbb{Q}$-acyclic. By Lemma 2.5 and Theorems 4.1 and 4.3, we may further assume that $Z(G)=1, O_{p^{\prime}}(G)=1$ and $G$ is not an almost-simple group.

On the other hand, by Theorem $4.5, \mathcal{A}_{p}(G)$ is not $\mathbb{Z}$-acyclic. In order to prove that it is not $\mathbb{Q}$-acyclic, it is enough to show that $\mathcal{A}_{p}(G)$ has free abelian homology. Since it has the homotopy type of a 2dimensional complex $K \subseteq \mathcal{K}\left(\mathcal{S}_{p}(G)\right)$, we only need to verify that $H_{n}\left(\mathcal{A}_{p}(G), \mathbb{Z}\right) \cong H_{n}(K, \mathbb{Z})$ is a free abelian group for $n=0,1,2$.

Clearly $H_{2}(K, \mathbb{Z})$ and $H_{0}(K, \mathbb{Z})$ are free abelian groups. Finally, by Theorem $4.2, \pi_{1}\left(\mathcal{A}_{p}(G)\right)$ is a free group, so its abelianisation $H_{1}(K, \mathbb{Z})$ is a free abelian group. This completes the proof.

We can extend Corollary 4.6 on the $p$-rank 3 case of $(\mathbb{Z}-Q C)$ to the rational version $(\mathbb{Q}-Q C)$.

Corollary 4.8. The rational Quillen's conjecture $(\mathbb{Q}-Q C)$ holds for groups of p-rank at most 3 .

\section{Particular cases}

In this section we prove Theorem 5.1, which allows us to eliminate components isomorphic to $\mathrm{L}_{2}\left(2^{3}\right)$ $(p=3), \mathrm{U}_{3}\left(2^{3}\right)(p=3)$ and $\mathrm{Sz}\left(2^{5}\right)(p=5)$ in a minimal counterexample to Quillen's conjecture. These simple groups were excluded as possible components during the analysis of the conjecture of Aschbacher and Smith [5], due to the particular structure of the centralisers of their field automorphisms of order $p$. Namely, these centralisers have nontrivial normal $p$-subgroups. However, we will see that because of this property, we can establish Quillen's conjecture for groups containing these types of components.

And since $\mathrm{Sz}\left(2^{5}\right)$ was the only obstruction in [5] for $p=5$, in particular we get their Main Theorem also for $p=5$. For that purpose, we will use the structure of these centralisers, which can be found in [8]. Recall that $R=\mathbb{Z}$ or $\mathbb{Q}$.

Theorem 5.1. Suppose that $G$ satisfies $(H 1)_{R}$ and contains a component $L$ such that $L / Z(L)$ is isomorphic to $\mathrm{L}_{2}\left(2^{3}\right)(p=3), \mathrm{U}_{3}\left(2^{3}\right)(p=3)$ or $\mathrm{Sz}\left(2^{5}\right)(p=5)$. Then $G$ satisfies $(R-Q C)$.

We summarise next the scheme of the proof of [5, Main Theorem] to see why these cases were excluded and to expose the key points to extend their theorem to every odd prime $p$. In particular, we include the proof of Corollary 3 during the discussion.

Let $p$ be an odd prime and $G$ a group of minimal order subject to failing (QQQC). Assume further that if $\mathrm{U}_{n}(q)$ is a component of $G$, with $q \equiv-1(\bmod p)$ and $q$ odd, then the $p$-extensions of $\mathrm{U}_{m}\left(q^{p^{e}}\right)$ satisfy $(\mathcal{D D})_{p}$ for all $m \leq n$ and $e \in \mathbb{Z}$. In particular, $G$ satisfies $(\mathrm{H} 1)_{\mathbb{Q}}$. The proof splits into three steps.

Step 1. We get $O_{p^{\prime}}(G)=1$ (this is [5, Proposition 1.6]).

To prove $O_{p^{\prime}}(G)=1$ in [5], Theorems 2.3 and 2.4 there are invoked. However, these theorems, stated for $p$ odd, require that $G$ not contain components isomorphic to $\mathrm{L}_{2}\left(2^{3}\right), \mathrm{U}_{3}\left(2^{3}\right)$ or $\mathrm{Sz}\left(2^{5}\right)$ with $p=3,3,5$, respectively. By our Theorem 4.1 , we can get the same reduction over $G$, since $(\mathrm{H} 1)_{\mathbb{Q}}$ holds, without those restrictions on $p$ and the components.

Step 2. If $L$ is a component of $G$, then some $p$-extension of $L$ fails $(2 \mathcal{D})_{p}$ (this is [5, Proposition 1.7]).

Similar to Step 1, [5, Theorems 2.3 and 2.4] are invoked here. By our Theorem 5.1, $G$ does not contain components isomorphic to $\mathrm{L}_{2}\left(2^{3}\right), \mathrm{U}_{3}\left(2^{3}\right)$ or $\mathrm{Sz}\left(2^{5}\right)$ with $p=3,3,5$, respectively. Therefore, 
we can invoke [5, Theorems 2.3 and 2.4] without those restrictions on $p$, so this step of the proof also extends to $p \geq 3$. The hypothesis on the unitary components also allows us to conclude that $G$ does not contain unitary components $\mathrm{U}_{n}(q)$ with $p \mid q+1$ and $q$ odd.

Step 3. $\tilde{H}_{*}\left(\mathcal{A}_{p}(G), \mathbb{Q}\right) \neq 0$, since $\tilde{\chi}\left(\mathcal{A}_{p}(G)^{g}\right) \neq 0$ for some $g \in G$.

First note that if $\tilde{H}_{*}\left(\mathcal{A}_{p}(G), \mathbb{Q}\right)=0$, then $\tilde{\chi}\left(\mathcal{A}_{p}(G)^{g}\right)=0$ for all $g \in G$, by the Lefschetz fixedpoint theorem. This step consists then of deriving a contradiction by showing that $\tilde{\chi}\left(\mathcal{A}_{p}(G)^{g}\right) \neq 0$ for some $g \in G$ [5, p. 490]. This is Robinson's method [17]. The idea is to look for a subgroup $Q=\langle g\rangle \times O_{2}(Q) \leq G$ with $O_{2}(Q) \neq 1$, for which $\tilde{\chi}\left(\mathcal{A}_{p}(G)^{g}\right) \equiv \tilde{\chi}\left(\mathcal{A}_{p}(G)^{Q}\right) \quad(\bmod 2)$ is nonzero. To construct such a subgroup $Q$, [5, Theorem 5.3] is invoked, which is stated for $p \geq 5$. Therefore, we can extend this step to $p=5$ and, consequently, [5, Main Theorem] to $p \geq 5$, but not to $p \geq 3$. This proves Corollary 3. (See also the comment after [5, Main Theorem] and the remark at the bottom of [5, p. 493]).

The extension of [5, Main Theorem] to $p=3$ will be treated in a forthcoming work. Roughly, if we want to construct such a subgroup $Q$ for $p=3$ in step 3, we need first to eliminate some possible components of $G$, as we are doing in Theorem 5.1.

The problem with the components $L \cong \mathrm{L}_{2}\left(2^{3}\right)(p=3), \mathrm{U}_{3}\left(2^{3}\right)(p=3)$ or $\mathrm{Sz}\left(2^{5}\right)(p=5)$ is that their $p$-extensions do not have nonconical complements, as defined in [5, Theorem 2.3], when they contain field automorphisms of order $p$. This implies that we cannot invoke [5, Theorem 2.4], which is needed to get hypothesis (v) of Lemma 3.14. Moreover, a $p$-extension $L A$ satisfies $(2 \mathcal{D})_{p}$ if and only if $A$ does not contain field automorphisms. In particular, if $G$ does not contain field automorphisms of order $p$ of these components, we can invoke [5, Theorem 2.4] in step 2 of the proof of [5, Main Theorem] and eliminate components of this stype. In consequence, if we want to proceed as in [5] and eliminate these components from a minimal counterexample $G$ to Quillen's conjecture, we only need to analyse the case where $G$ contains a $p$-extension $L A$ with $A$ including field automorphisms of order $p$.

The obstruction relies on the fact that $O_{p}\left(C_{L}(A)\right) \neq 1$ for every $A$ inducing field automorphisms of order $p$ on such $L$. Nevertheless, this special feature is exactly the ingredient we want to construct a proper subposet $X \subset \mathcal{A}_{p}(G)$ suitable for use as $X$ in Lemma 3.14. This key property allows us to extract these subgroups $A$ from the poset $\mathcal{A}_{p}(G)$ and apply Lemma 3.14 with $H=L$.

Before we proceed with the proof of Theorem 5.1, we describe some preliminary results that will be repeatedly used from now on.

Remark 5.2. With the aim of showing that $\tilde{H}_{*}\left(\mathcal{A}_{p}(G)\right) \neq 0$, we will look for a subposet $X \subseteq \mathcal{A}_{p}(G)$ and subgroups $H, K$ to fit in the hypotheses of Lemma 3.14. We will also require $X$ homotopy-equivalent to $\mathcal{A}_{p}(G)$. Indeed, we will construct $X, H$ and $K$ from a very particular component $L$ of $G$. In general, we will pick $H=L A$ and $K=C_{G}(L A)$, where $A \leq N_{G}(L)$ is faithful on the component $L$. Our first observation is the following:

(0) We will choose $A \in \mathcal{A}_{p}(G)$ such that $A \cap L \neq 1$, so $A \leq N_{G}(L)$ if $Z(L)$ is a $p^{\prime}$-group.

Note that if $A \in \mathcal{A}_{p}(G)$ and $1 \neq A \cap L$, then $1 \neq A \cap L \leq L \cap L^{a}$ for all $a \in A$. When $Z(L)$ is a $p^{\prime}$-group, this forces $L=L^{a}$ and $A \leq N_{G}(L)$.

(1) In proving $(\mathbb{Q}-\mathrm{QC})$, we will assume $O_{p}(G)=1$. Hence $O_{p}(L)=1$, so $Z(L)$ is a $p^{\prime}$-group. Therefore, when $H=L$ we get hypothesis (i) of Lemma 3.14.

(2) In proving $(\mathbb{Q}-\mathrm{QC})$, we can also assume $(\mathrm{H} 1)_{\mathbb{Q}}$ - for example, by studying a minimal counterexample.

(3) With $(\mathrm{H} 1)_{\mathbb{Q}}$, by Theorem 4.1 we have the reduction $O_{p^{\prime}}(G)=1$. Hence with $O_{p}(G)=1$, we get

$$
Z(E(G)) \leq Z\left(F^{*}(G)\right)=C_{G}\left(F^{*}(G)\right) \leq F(G) \leq O_{p}(G) O_{p^{\prime}}(G)=1 .
$$


Therefore, using (2) - that is, (H1) - for $H=L$ and $K=C_{G}(L)$ we get hypothesis (v) of Lemma 3.14. Note that in general, if $L$ is a component of $G$ and $F(G)=1$, then $O_{p}\left(C_{G}(L)\right)=1$ and $Z(L)=1$.

To conclude this remark, we provide some possible paths to use Lemma 3.14.

Case 1. $H=L, K=C_{G}(L)$.

In particular, we automatically get hypotheses (i) and (v) of Lemma 3.14 by (1) and (3).

Subcase 1a. $X=\mathcal{A}_{p}(G)$.

Here we get Lemma 3.14(a) and (b), so we only need to establish (c). That is, take $A$ exhibiting $(Q \mathcal{D})_{p}$ for $L$ with $\mathcal{A}_{p}(G)_{>A} \subseteq A \times K$.

This will hold for example in case 1 of the proof of Theorems 5.1 and 6.1. There we do get $(Q \mathcal{D})_{p}$ for $L$, and 'no outers' says roughly that any $A$ maximal in $L$ is in fact maximal faithful on $L$, so every $E \in \mathcal{A}_{p}(G)_{>A}$ has the form $E=A C_{E}(L) \subseteq A \times K$.

Subcase 1b. $X \subsetneq \mathcal{A}_{p}(G)$.

Here we will have more work to do, for example, establishing conditions (ii)-(iv) of Lemma 3.14, and of course showing that $X$ and $\mathcal{A}_{p}(G)$ are homotopy-equivalent.

Case 2. $H=L A>L, K=C_{G}(L A)$.

In this case, we will typically begin by constructing $H$ as $L E$, where $E$ does not contain inner automorphisms of $L$, and later we will choose $A \in \mathcal{A}_{p}(L E)$ with $L A=L E$.

Denote by $\operatorname{Inn}(G)$ the subgroup of $\operatorname{Aut}(G)$ of inner automorphisms of $G$. If $\phi \in \operatorname{Aut}(G)-\operatorname{Inn}(G)$, we say that $\phi$ induces an outer automorphism on $G$. If $L \leq G$ and $E \leq N_{G}(L)$, then we can describe the types of automorphisms (inner or outer) induced by the action of $E$ on $L$ via the map $E \rightarrow \operatorname{Aut}(L)$.

Lemma 5.3. Let $L \leq G$ and $E \leq N_{G}(L)$. Then

$$
E \cap\left(L C_{G}(L)\right)=\{x \in E: x \text { induces an inner automorphism on } L\} .
$$

In particular, $E \cap\left(L C_{G}(L)\right)=1$ if and only if $E$ acts by outer automorphisms on $L$.

Proof. Clearly $E \cap\left(L C_{G}(L)\right)$ acts by inner automorphisms on $L$. If $x \in E$ induces an inner automorphism on $L$, then there exists $y \in L$ such that $z=y^{-1} x$ acts trivially on $L$. Therefore, $z \in C_{G}(L)$ and $x=y z \in L C_{G}(L)$.

Remark 5.4. If $H \leq G$ and $m_{p}(H)=m_{p}(G)=m$, then $\tilde{H}_{m-1}\left(\mathcal{A}_{p}(H)\right) \subseteq \tilde{H}_{m-1}\left(\mathcal{A}_{p}(G)\right)$. In particular, if $H$ has $(\mathcal{D D})_{p}$ then so does $G$.

On the other hand, if $L=L_{1} \times \cdots \times L_{n}$ is a direct product and each $L_{i}$ has $(Q \mathcal{D})_{p}$, then $L$ has $(\mathcal{D D})_{p}$. This follows from Proposition 2.3 and the homology decomposition of a join.

The following lemma follows from the $p$-rank 2 case of the conjecture [16, Proposition 2.10].

Lemma 5.5. Suppose that $O_{p}(G)=1$. If $m_{p}(G)=1$, or $m_{p}(G)=2$ with $\mathcal{A}_{p}(G)$ connected, then $G$ has $(\mathcal{Q D})_{p}$.

Proof. By [16, Proposition 2.10], $\tilde{H}_{*}\left(\mathcal{A}_{p}(G)\right) \neq 0$. If $m_{p}(G)=1$, then clearly we have $\tilde{H}_{0}\left(\mathcal{A}_{p}(G)\right) \neq$ 0 . If $m_{p}(G)=2$ and $\mathcal{A}_{p}(G)$ is connected, then $\tilde{H}_{0}\left(\mathcal{A}_{p}(G)\right)=0$, so $\tilde{H}_{1}\left(\mathcal{A}_{p}(G)\right) \neq 0$.

Now we prove Theorem 5.1.

Proof of Theorem 5.1. We prove the rational version $(\mathbb{Q}-\mathrm{QC})$. That is, we show that $O_{p}(G)=1 \mathrm{implies}$ $\tilde{H}_{*}\left(\mathcal{A}_{p}(G), \mathbb{Q}\right) \neq 0$. The same proof works for the integer version (ZZ-QC), by Remark 6.2. 
As we are proving $(\mathbb{Q}-\mathrm{QC})$, we have its hypothesis $O_{p}(G)=1$. Let $L$ be a component of $G$ as in the hypotheses of the theorem. The preliminary Remark 5.2 shows then that we can suppose that $O_{p^{\prime}}(G)=1$, so $Z(L)=1$ and $L \cong \mathrm{L}_{2}\left(2^{3}\right), \mathrm{U}_{3}\left(2^{3}\right)$ or $\mathrm{Sz}\left(2^{5}\right)$, with $p=3,3$ or 5 , respectively. We use the structure of the centralisers of the automorphisms of $L$. We refer to [8, Part I, (7-2), (9-1) and (9-3)] for more details on the following assertions.

○ If $L=\mathrm{L}_{2}\left(2^{3}\right)$, then $\operatorname{Aut}(L) \cong L \rtimes\langle\phi\rangle$, where $\phi$ induces a field automorphism of order 3 on $L$, and $C_{L}(\phi) \cong \mathrm{L}_{2}(2) \cong \mathbb{S}_{3} \cong C_{3} \rtimes C_{2}$. Note that $m_{p}(L)=1$.

○ If $L=\mathrm{U}_{3}\left(2^{3}\right)$, then $\operatorname{Out}(L)=\operatorname{Out} \operatorname{diag}(L) \rtimes C_{6}$ and $\operatorname{Out} \operatorname{diag}(L) \cong C_{3}$. If $\phi \in \operatorname{Aut}(L)$ is a field automorphism of order 3 , then $C_{\operatorname{Inn} \operatorname{diag}(L)}(\phi)=C_{L}(\phi) \cong \mathrm{PGU}_{3}(2) \cong\left(\left(C_{3} \times C_{3}\right) \rtimes Q_{8}\right) \rtimes C_{3}$.

(A) In particular, field automorphisms and diagonal automorphisms do not commute, so any purely outer automorphism group of $\mathrm{U}_{3}\left(2^{3}\right)$ has 3-rank at most 1 .

(B) Note that $m_{p}(L)=2$ and $\mathcal{A}_{p}(L)$ is connected, since it does not appear in the disconnected list of Theorem A.1.

○ If $L=\operatorname{Sz}\left(2^{5}\right)$, then $\operatorname{Aut}(L) \cong L \rtimes\langle\phi\rangle$, where $\phi$ induces a field automorphism of order 5 on $L$, and $C_{L}(\phi) \cong \mathrm{Sz}(2) \cong C_{5} \rtimes C_{4}$. Note that $m_{p}(L)=1$.

In any case, $L$ satisfies $(\mathcal{D D})_{p}$ by Lemma 5.5, and if $\phi \in N_{G}(L)$ induces a field automorphism of order $p$ on $L$, then $O_{p}\left(C_{L}(\phi)\right) \neq 1$. Let $\mathcal{N}=\left\{E \in \mathcal{A}_{p}\left(N_{G}(L)\right): E \cap\left(L C_{G}(L)\right) \neq 1\right\}$.

We are going to use some of the claims stated in Remark 5.2. In particular, we get $O_{p}\left(C_{G}(L)\right)=1$ by item (3) of that remark.

We split the remainder of the proof into two cases.

Case 1: $\mathcal{A}_{p}\left(N_{G}(L)\right)=\mathcal{N}$.

We are in subcase 1a of Remark 5.2. That is, we take $X=\mathcal{A}_{p}(G), H=L$ and $K=C_{G}(L)$, which satisfy hypothesis (v) and conditions (a) and (b) of Lemma 3.14.

Recall that we observed earlier that $H=L$ satisfies $(Q \mathcal{D})_{p}$, which is exhibited by some $A \in \mathcal{A}_{p}(L)$. The hypothesis $\mathcal{A}_{p}\left(N_{G}(L)\right)=\mathcal{N}$ of this case implies that there is no $E \in \mathcal{A}_{p}(G)$ inducing only outer automorphisms on $L$. Therefore, if $E \in \mathcal{A}_{p}(G)_{>A}$, then $E=A C_{E}(L) \subseteq A \times K$. This shows that condition (c) of Lemma 3.14 holds, and therefore we conclude that $\tilde{H}_{*}\left(\mathcal{A}_{p}(G), \mathbb{Q}\right) \neq 0$ in this case.

Case 2: $\mathcal{A}_{p}\left(N_{G}(L)\right) \neq \mathcal{N}$.

Here, every $E \in \mathcal{A}_{p}\left(N_{G}(L)\right)-\mathcal{N}$ induces outer automorphisms on $L$ (see Lemma 5.3). In conjunction with item (A) regarding the unitary group $\mathrm{U}_{3}\left(2^{3}\right)$, we conclude that $|E|=p$.

Next we show that if $E$ induces field automorphisms on $L$, then $\mathcal{A}_{p}(G)_{>E}$ is contractible. Recall that the existence of field automorphisms of order $p$ in $G$ for components of this type is the real obstruction in [5] to handling these components. The idea here is then to extract these elements $E$ from $\mathcal{A}_{p}(G)$ to get a homotopy-equivalent subposet $X$ which will be more suitable for applying Lemma 3.14 (see also subcase 1 b of Remark 5.2).

Claim. If $E \in \mathcal{A}_{p}\left(N_{G}(L)\right)$ is a group of purely outer automorphisms of $L$ containing field automorphisms of order $p$, then $\mathcal{A}_{p}(G)_{>E}$ is contractible.

Proof. Let $E$ be as in the hypothesis of the claim. Then $|E|=p$, and $O_{p}\left(C_{L}(E)\right) \neq 1$. We show that $\mathcal{A}_{p}(G)_{>E}$ is contractible by exhibiting a sequence of homotopy equivalences.

Take $M=C_{L}(E) C_{G}(L E)$. Since $E$ induces only outer automorphisms on $L$ and $M$ contains only inners, we have

$$
E \cap M=1 .
$$

We will prove that

$$
\mathcal{A}_{p}(G)_{>E} \simeq Y \simeq \mathcal{A}_{p}(M) \simeq *,
$$


where

$$
Y:=\left\{B \in \mathcal{A}_{p}(G): N_{B}(L)>E\right\} \subseteq \mathcal{A}_{p}(G)_{>E} .
$$

In order to prove that $\mathcal{A}_{p}(G)_{>E} \simeq Y$, we show that $Y_{>B}$ is contractible for each $B \in \mathcal{A}_{p}(G)_{>E}-Y$, and then conclude that the inclusion $Y \hookrightarrow \mathcal{A}_{p}(G)_{>E}$ is a homotopy equivalence by Proposition 2.2. To that aim, we construct a homotopy equivalence $Y_{>B} \simeq \mathcal{A}_{p}\left(C_{M}(B)\right)$, and then we prove that $O_{p}\left(C_{M}(B)\right) \neq 1$.

Fix $B \in \mathcal{A}_{p}(G)_{>E}-Y$ and take $C \in Y_{>B}$. We prove that $N_{C}(L) \cap M \neq 1$, and in particular $C \cap M \neq 1$. If $N_{C}(L)$ contains inner automorphisms of $L$ or acts nonfaithfully on $L$, then $N_{C}(L) \cap M \neq 1$ by Lemma 5.3. If $N_{C}(L)$ acts faithfully on $L$ and it does not contain inner automorphisms of $L$, then $N_{C}(L)$ embeds into both $\operatorname{Aut}(L)$ and $\operatorname{Out}(L)$, and it has $p$-rank at least 2 since $E<N_{C}(L)$. However, $N_{C}(L)$ can only contain field automorphisms of $L$, since diagonal and field automorphisms (of order $p$ ) do not commute (see (A) earlier). On the other hand, a subgroup of $\operatorname{Out}(G)$ containing only field automorphisms of $L$ is cyclic of order $p$, showing that $N_{C}(L)$ has $p$-rank at most 1 . We have a well-defined homotopy equivalence $C \in Y_{>B} \mapsto C \cap M \in \mathcal{A}_{p}\left(C_{M}(B)\right)$. The inverse is given by $C \in \mathcal{A}_{p}\left(C_{M}(B)\right) \mapsto C B$. Note that $C B \in Y_{>B}$, since $E \cap C \leq E \cap M=1$ by equation (5.1), so

$$
C B \geq N_{C B}(L) \geq E C>E .
$$

Therefore, $Y_{>B} \simeq \mathcal{A}_{p}\left(C_{M}(B)\right)$.

Next we prove that $Y_{>B}$ is contractible by showing that $O_{p}\left(C_{M}(B)\right) \neq 1$. Decompose $B=E \tilde{B}$, where $\tilde{B}$ is a complement to $E$ in $B$. Since $N_{B}(L)=E, \tilde{B}$ acts regularly on the set $\left\{L^{b}: b \in \tilde{B}\right\}$. Let $K=\left\langle L^{b}: b \in \tilde{B}\right\rangle$. It is not hard to see that $C_{K}(B) \cong C_{L}(E)$. Finally, observe that $C_{K}(B)$ is a normal subgroup of $C_{M}(B)$, so $O_{p}\left(C_{M}(B)\right) \neq 1$. Therefore, $Y_{>B} \simeq \mathcal{A}_{p}\left(C_{M}(B)\right)$ is contractible. By Proposition 2.2, $Y \hookrightarrow \mathcal{A}_{p}(G)_{>E}$ is a homotopy equivalence.

By taking $B=1$ in the foregoing reasoning, $Y \simeq \mathcal{A}_{p}(M)$ is contractible, since $1 \neq O_{p}\left(C_{L}(E)\right) \leq$ $O_{p}\left(C_{L}(E) C_{G}(L E)\right)=O_{p}(M)$. In consequence, $\mathcal{A}_{p}(G)_{>E} \simeq Y$ is contractible. This finishes the proof of this claim.

Now we construct our subposet $X$ homotopy-equivalent to $\mathcal{A}_{p}(G)$, which is roughly obtained from $\mathcal{A}_{p}(G)$ by removing any $E \in \mathcal{A}_{p}\left(N_{G}(L)\right)$ faithful on $L$ and containing some field automorphism of order $p$. To this end, we will actually concatenate two homotopy equivalences. In particular, if there are no field automorphisms of order $p$ of $L$ in $N_{G}(L)$, then $X=\mathcal{A}_{p}(G)$.

We construct the first homotopy equivalence by extracting the purely outers that contain field automorphisms of $L$. That is, in combination with Proposition 2.2 and the foregoing claim, the subposet

$$
X_{0}=\left\{E \in \mathcal{A}_{p}(G): \text { if }|E|=p \text {, then it does not induce field automorphisms on } L\right\}
$$

is homotopy-equivalent to $\mathcal{A}_{p}(G)$.

For the second homotopy equivalence, we extract the remaining elementary abelian $p$-subgroups acting faithfully on $L$ and containing some field automorphisms. Let

$$
W=\left\{E \in \mathcal{A}_{p}\left(N_{G}(L)\right): C_{E}(L)=1 \text { and } E \text { contains some field automorphism of } L\right\}
$$

and

$$
X=\mathcal{A}_{p}(G)-W
$$

If $E \in X_{0}-X$, then $E$ is faithful on $L$ and it contains some field automorphisms of order $p$ of $L$, but $|E|>p$ since $E \in X_{0}$. On the other hand, since field and diagonal automorphisms do not commute in the unitary case by item (A), we see that $E$ contains inner automorphisms of $L$. That is, $1 \neq E \cap\left(L C_{G}(L)\right)$ by Lemma 5.3, and moreover, $\left|E: E \cap\left(L C_{G}(L)\right)\right|=p$. Hence, $X_{<E}=\mathcal{A}_{p}\left(E \cap\left(L C_{G}(L)\right)\right) \simeq *$. By Proposition 2.2, $X \simeq X_{0} \simeq \mathcal{A}_{p}(G)$. 
Note that if we do not have field automorphisms of order $p$ in $N_{G}(L)$ but we are in case 2, then we have no points to remove from $\mathcal{A}_{p}(G)$ and $X=\mathcal{A}_{p}(G)$. Moreover, the only possibility here is $L=\mathrm{U}_{3}\left(2^{3}\right)$ and $p=3$, with $N_{G}(L)$ containing diagonal automorphisms of order 3 , since $\mathcal{A}_{p}\left(N_{G}(L)\right) \neq \mathcal{N}$. Since both Inn diag $\left(\mathrm{U}_{3}\left(2^{3}\right)\right)$ and $\mathrm{U}_{3}\left(2^{3}\right)$ have 3-rank 2 and the latter satisfies $(Q \mathcal{D})_{p}$, by Remark 5.4 Inn diag $\left(\mathrm{U}_{3}\left(2^{3}\right)\right)$ also satisfies $(2 \mathcal{D})_{p}$, exhibited by cycles of $\mathcal{A}_{3}\left(\mathrm{U}_{3}\left(2^{3}\right)\right)$.

In any scenario, to conclude the proof of case 2, apply Lemma 3.14 with the obtained subposet $X$, $H=L, K=C_{G}(L)$ and $a \in \mathcal{A}_{p}(L)^{\prime}$ any chain exhibiting $(Q \mathcal{D})_{p}$ for $L$. Note that we get the inclusion $X_{>\max a} \subseteq \mathcal{N}_{K}$, since we removed the elements of $\mathcal{A}_{p}(G)$ that contain field automorphisms of $L$, and $\max a \leq L$ is maximal faithful on $L$.

\section{Components of $p$-rank 1}

In this section we show that under $(\mathrm{H} 1)_{R}$, if $G$ has a component of $p$-rank 1 , then $G$ satisfies $(R$-QC), with $R=\mathbb{Z}$ or $\mathbb{Q}$ (see Theorem 6.1). We refer to [8, Part I] for the main properties of simple groups of $p$-rank 1. Recall that there are no simple groups of 2-rank 1.

Theorem 6.1. Suppose that $G$ satisfies $(H 1)_{R}$ and contains a component $L$ such that $L / Z(L)$ has p-rank 1. Then $G$ satisfies $(R-Q C)$.

Proof. We proceed similarly to Theorem 5.1, so following Remark 5.2 we can suppose that $O_{p}(G)=$ $1=O_{p^{\prime}}(G)$, and we get $O_{p}\left(C_{G}(L)\right)=1$ and $Z(L)=1$. Hence $L$ is a simple group of $p$-rank 1 and $p$ is odd. Recall also that if $B \in \mathcal{A}_{p}(G)$ and $B \cap L \neq 1$, then $B \leq N_{G}(L)$.

Let $\mathcal{N}=\left\{E \in \mathcal{A}_{p}\left(N_{G}(L)\right): E \cap\left(L C_{G}(L)\right) \neq 1\right\}$. We split the proof into two cases.

Case 1. $\mathcal{A}_{p}\left(N_{G}(L)\right)=\mathcal{N}$.

In this case, there are no outer automorphisms of order $p$ of $L$ inside $G$, and $\Omega_{1}\left(N_{G}(L)\right) \cong$ $L \times \Omega_{1}\left(C_{G}(L)\right)$. Set $A \in \mathcal{A}_{p}(L)$. Since $L$ has $p$-rank $1, A$ is maximal faithful on $L$ and represents a connected component of $\mathcal{A}_{p}(L)$ exhibiting $(Q \mathcal{D})_{p}$ for $L$ (see Lemma 5.5). Moreover, by item (0) in Remark 5.2, we get $\mathcal{A}_{p}(G)_{>A} \subseteq A \times C_{G}(L)$. The hypotheses of Lemma 3.14 are verified with $H=L$, $K=C_{G}(L), a=(A) \in \alpha \in \tilde{C}_{0}\left(\mathcal{A}_{p}(L)\right)$ and $X=\mathcal{A}_{p}(G)$. This finishes the proof of case 1 .

In following case, we will see that in some subcases we take $H=L A>L$ and we do not remove points, so we apply Lemma 3.14 with $X=\mathcal{A}_{p}(G)$.

Case 2. $\mathcal{A}_{p}\left(N_{G}(L)\right) \neq \mathcal{N}$.

Note that every $E \in \mathcal{A}_{p}\left(N_{G}(L)\right)-\mathcal{N}$ induces outer automorphisms on $L$ and has order $p$, since $m_{p}(L)=1$ (see Table 1). By Theorem 5.1, we can suppose that $L$ is not isomorphic to $\mathrm{L}_{2}\left(2^{3}\right)(p=3)$ nor to $\mathrm{Sz}\left(2^{5}\right)(p=5)$. Therefore, $m_{p}(L E)=2$ and $L E$ has $(Q \mathcal{D})_{p}$ (i.e., it is connected; see Table 1$)$.

Case 2a. There exists $E \in \mathcal{A}_{p}\left(N_{G}(L)\right)-\mathcal{N}$ with $O_{p}\left(C_{G}(L E)\right)=1$.

Take such an element $E$ and let $K=C_{G}(L E)$. Note that hypotheses (v), (a) and (b) of Lemma 3.14 hold with $X=\mathcal{A}_{p}(G), H=L E$ and $K=C_{G}(L E)$. We show how to get hypothesis (c).

Pick $A \in \mathcal{A}_{p}(L E)$ of order $p^{2}$ exhibiting $(\mathcal{D D})_{p}$ for $L E$, and observe that $L A=L E$ and $|A \cap L|=$ p. If $B \in \mathcal{A}_{p}(G)_{>A}$, then $B \cap L \neq 1$ and hence $B \in \mathcal{A}_{p}\left(N_{G}(L)\right)$. Moreover, $C_{B}(L) \neq 1$ since $A \leq B / C_{B}(L) \leq \operatorname{Aut}(L)$, and the latter has $p$-rank 2. In consequence, $B=A C_{B}(L)$. This shows that $\mathcal{A}_{p}(G)_{>A} \subseteq A \times K$, and for some 1-cycle $\alpha$ and chain $a \in \mathcal{A}_{p}(L A)^{\prime}$, we have $A \in a \in \alpha \in \tilde{C}_{1}\left(\mathcal{A}_{p}(L A)\right)$ exhibiting $(2 \mathcal{D})_{p}$ for $H$. Hence hypothesis (c) of Lemma 3.14 holds and we get $\tilde{H}_{*}\left(\mathcal{A}_{p}(G), \mathbb{Q}\right) \neq 0$. This concludes the proof of case $2 \mathrm{a}$.

Case 2b. For all $E \in \mathcal{A}_{p}\left(N_{G}(L)\right)-\mathcal{N}$ we have $O_{p}\left(C_{G}(L E)\right) \neq 1$.

Here we show that the poset $X$ to be defined in equation (6.1) is a homotopy-equivalent and proper subposet of $\mathcal{A}_{p}(G)$ that fits in the hypotheses of Lemma 3.14 with $H=L$ and $K=C_{G}(L)$.

Consider the subposets $\mathcal{F}_{0}=\mathcal{A}_{p}\left(N_{G}(L)\right)-\mathcal{N}, \mathcal{F}_{1}=\left\{E \in \mathcal{A}_{p}\left(N_{G}(L)\right):|E|=p^{2}\right.$, $\left.C_{E}(L)=1, E \cap L \neq 1\right\}$ and

$$
X:=\mathcal{A}_{p}(G)-\mathcal{F}_{1} .
$$


We show that $X \simeq \mathcal{A}_{p}(G)$. If $E \in \mathcal{F}_{1}$, then $E=(E \cap L) E_{0}$, where $E_{0} \cap\left(L C_{G}(L)\right)=1$. Hence $E_{0} \in \mathcal{F}_{0}$ and $O_{p}\left(C_{G}(L E)\right)=O_{p}\left(C_{G}\left(L E_{0}\right)\right) \neq 1$. Let $B \in X_{>E}$. Since $m_{p}(\operatorname{Aut}(L))=2$ and $E$ acts faithfully on $L$ with $B \cap L \geq E \cap L \neq 1$, we have $B \leq N_{G}(L), C_{B}(L) \neq 1$ and $B=E C_{B}(L)$. Therefore, $X_{>E} \simeq \mathcal{A}_{p}\left(C_{G}(L E)\right)$, where the homotopy equivalence is given by $B \mapsto C_{B}(L)$ with inverse $C \mapsto C E$. By Proposition 2.2, $X \simeq \mathcal{A}_{p}(G)$.

Finally, we appeal to Lemma 3.14 on this subposet $X$, with $H=L, K=C_{G}(L)$ and $a=(A) \in \alpha \in$ $\tilde{C}_{0}\left(\mathcal{A}_{p}(L)\right)$, where $A \in \mathcal{A}_{p}(L)$. This concludes the proof of case $2 \mathrm{~b}$.

In any case, we have shown that $G$ satisfies $(\mathbb{Q}-Q C)$. For the integer version $(\mathbb{Z}-Q C)$ of this case, see Remark 6.2.

Remark 6.2. In the proofs of Theorems 5.1 and 6.1, we invoked Lemma 3.14 with some cycle $\alpha$ containing an arbitrary full chain $a$. Note that we could have chosen first $\alpha$ and then $a \in \alpha$ in these proofs. Since $\alpha$ is an element of either $\tilde{C}_{1}(X)$ or $\tilde{C}_{0}(X)$, it can be taken to have coefficients equal to \pm 1 . For example, if $\alpha \in \tilde{C}_{1}(X)$, then pick $\alpha$ to be a simple cycle (that is, a cycle in the 1 -skeleton of the simplicial complex that does not self-intersect). By Remark 3.15, Theorems 5.1 and 6.1 extend to the integer version of the conjecture (ZZ-QC).

\section{The $p$-rank 4 case of Quillen's conjecture}

In this section we prove Theorem 4, establishing (Q-QC) for groups of $p$-rank at most 4 . We use the results of the previous sections together with the classification of finite groups with a strongly $p$ embedded subgroup. We provide in Appendix A further details of this classification, as well as some properties of these groups. We will see that the structure of the centralisers of the simple groups of low $p$-rank plays a fundamental role in the proof of this theorem.

The following elementary remark will be useful in the proof of Theorem 4.

Remark 7.1. Suppose that $L$ is a normal subgroup of $G$ such that $Z(L)$ is a $p^{\prime}$-group (e.g., when $L$ is a normal component of $G$ ). If every order $p$ element of $G$ induces an inner automorphism on $L$, then $\Omega_{1}(G) \leq L C_{G}(L)$ by Lemma 5.3. In particular, $\mathcal{A}_{p}(G)=\mathcal{A}_{p}\left(\Omega_{1}(G)\right) \simeq \mathcal{A}_{p}(L) * \mathcal{A}_{p}\left(C_{G}(L)\right)$ by Propositions 2.3 and 2.4.

Proof of Theorem 4. Let $G$ be a group of $p$-rank at most 4 and suppose that $O_{p}(G)=1$. We prove that $\tilde{H}_{*}\left(\mathcal{A}_{p}(G), \mathbb{Q}\right) \neq 0$. Without loss of generality, we can assume that $G$ satisfies $(\mathrm{H} 1)_{\mathbb{Q}}$, and hence that the following conditions hold:

1. $G=\Omega_{1}(G)$ and $Z(G)=1$ (by Lemma 2.5).

2. $m_{p}(G)=4$ (by Corollary 4.8 ).

3. $O_{p^{\prime}}(G)=1$ (by Theorem 4.1).

4. $F^{*}(G)=L_{1} \cdots L_{n}$ is the direct product of simple components $L_{i}$ of order divisible by $p$ (by Remark 2.1).

5. $G$ is not an almost-simple group (by Theorem 4.3).

6. Every component of $G$ has $p$-rank at least 2 (by Theorem 6.1).

Since $G$ is not almost simple, $n \geq 2$. By condition (6), $m_{p}\left(L_{i}\right) \geq 2$ for all $i$, and since $4 \geq m_{p}\left(F^{*}(G)\right) \geq$ $2 n$, we conclude that $n=2, m_{p}\left(L_{1}\right)=2=m_{p}\left(L_{2}\right)$ and $m_{p}\left(F^{*}(G)\right)=4$.

If both $\mathcal{A}_{p}\left(L_{1}\right)$ and $\mathcal{A}_{p}\left(L_{2}\right)$ are connected, then $L_{1}$ and $L_{2}$ have $(Q \mathcal{D})_{p}$ by Lemma 5.5 , and so do $F^{*}(G)$ and $G$ by Remark 5.4. Indeed, more generally, this argument shows the following case.

Case 0. If $G$ contains a direct product of distinct subgroups $G_{1}, G_{2}$ of $p$-rank 2 for which $\mathcal{A}_{p}\left(G_{1}\right)$ and $\mathcal{A}_{p}\left(G_{2}\right)$ are connected with $O_{p}\left(G_{i}\right)=1, i=1,2$, then $\tilde{H}_{*}\left(\mathcal{A}_{p}(G), \mathbb{Q}\right) \neq 0$.

In consequence, we can suppose that $\mathcal{A}_{p}\left(L_{1}\right)$ is disconnected - that is, that $L_{1}$ has a strongly $p$ embedded subgroup. The possibilities for such an $L_{1}$ are described in Theorem A.1. In particular, if $p=2$ then $L_{1}$ is isomorphic either to $\mathrm{L}_{2}\left(2^{2}\right) \cong \mathbb{A}_{5}$ or to $\mathrm{U}_{3}\left(2^{2}\right)$ by a $p$-rank argument (see Table 1 ). 
On the other hand, by Remark 7.1, if $G$ has a normal component $L_{i}$ then we can suppose that $G$ contains an outer automorphism of $L_{i}$ of order $p$, so $p|| \operatorname{Out}\left(L_{i}\right) \mid$. Therefore, if $p$ is odd, both $L_{i}$ are normal in $G$, and this forces $p=3$ and $L_{1} \cong \mathrm{L}_{3}\left(2^{2}\right)$ by Table 1 .

Case 1. $p=2$ and $L_{1} \cong \mathbb{A}_{5}$ or $\mathrm{U}_{3}\left(2^{2}\right)$.

If $f$ is an outer involution of $\mathbb{A}_{5}$ (resp., $\left.\mathrm{U}_{3}\left(2^{2}\right)\right)$, then $C_{\mathbb{A}_{5}}(f) \cong \mathbb{S}_{3}$ of 2-rank 1 (resp., $C_{\mathrm{U}_{3}\left(2^{2}\right)}(f) \cong \mathbb{A}_{5}$ of 2-rank 2). Both centralisers have disconnected Quillen poset at $p=2$. Moreover, $\operatorname{Aut}\left(\mathbb{A}_{5}\right)=\mathbb{S}_{5} \cong \mathbb{A}_{5} \rtimes C_{2}$ and Aut $\left(\mathrm{U}_{3}\left(2^{2}\right)\right)=\mathrm{U}_{3}\left(2^{2}\right) \rtimes C_{4}$, with $C_{4}$ inducing field automorphisms on $\mathrm{U}_{3}\left(2^{2}\right)$.

We split the proof into two cases: when $L_{1}, L_{2}$ are permuted and when they are normal in $G$.

Case 1a. Some involution $x \in G$ permutes $L_{1}$ with $L_{2}$.

Then $N_{G}\left(L_{1}\right)=N_{G}\left(L_{2}\right)$ and it is a normal subgroup of $G$ of $p$-rank 4 with $G=N_{G}\left(L_{1}\right) T$, where $T=\langle x\rangle$. If $N_{G}\left(L_{1}\right)$ induces no outer automorphism of order 2 on $L_{1}$, then $N_{G}\left(L_{1}\right)=L_{1} \times L_{2}$, and hence $G \cong L_{1}$ < $C_{2}$. In this case, $\pi_{1}\left(\mathcal{A}_{2}(G)\right)$ is a nontrivial free group by [12, Theorem 5.6], and therefore $\tilde{H}_{1}\left(\mathcal{A}_{2}(G), \mathbb{Q}\right) \neq 0$.

Now assume that $N_{G}\left(L_{1}\right)$ induces some outer automorphism, say $f$, of order 2 on $L_{1}$. This eliminates $L_{1} \cong \mathrm{U}_{3}\left(2^{2}\right)$, since $C_{L_{1}}(f)$ has 2-rank 2, which implies $m_{2}\left(N_{G}\left(L_{1}\right)\right) \geq 5$. Hence $L_{1} \cong \mathbb{A}_{5}$. Now, $\mathbb{A}_{5}\langle f\rangle \cong \mathbb{S}_{5}$, which has 2-rank 2 and $(\mathcal{D D})_{2}$ (since $\mathcal{A}_{2}\left(\mathbb{S}_{5}\right)$ is connected). By case $0, N_{G}\left(L_{1}\right)=$ $\left(L_{1} \times L_{2}\right)\langle f\rangle$ and $f$ induces an outer automorphism on both $L_{1}$ and $L_{2}$. Then the subposet

$$
\mathfrak{i}\left(\mathcal{A}_{2}(G)\right):=\left\{E \in \mathcal{A}_{2}(G): E \text { is the intersection of maximal elements of } \mathcal{A}_{2}(G)\right\}
$$

has dimension 2 (rather than the dimension 3 of $\mathcal{A}_{2}(G)$ itself). This can be proved by using a similar argument to that of [14, Examples 4.10 and 4.11]. Finally, Corollary 4.7 applies since $\mathcal{K}\left(\mathfrak{i}\left(\mathcal{A}_{2}(G)\right)\right)$ is a $G$-invariant subcomplex of $\mathcal{K}\left(\mathcal{S}_{2}(G)\right)$ and homotopy-equivalent to $\mathcal{K}\left(\mathcal{A}_{2}(G)\right)$. This finishes the proof of case 1a.

Case 1b. $L_{1}$ is normal in $G$ (hence $L_{2}$ is also normal in $G$ ).

By Remark 7.1, we may assume the following:

(H2) Both components $L_{1}$ and $L_{2}$ admit nontrivial outer automorphisms from $G$.

Let $H=L_{1} C_{G}\left(L_{1}\right)$ and define $\mathcal{N}:=\mathcal{N}(H)$ and $\mathcal{F}:=\mathcal{A}_{2}(G)-\mathcal{N}$. By $(\mathrm{H} 2), \mathcal{F}$ is nonempty, and by Lemma 2.9 we can suppose that some $E \in \mathcal{F}$ has $1=O_{2}\left(C_{H}(E)\right)=O_{2}\left(C_{L_{1}}(E)\right) O_{2}\left(C_{G}\left(L_{1} E\right)\right)$. Note that the elements of $\mathcal{F}$ have order 2.

Subcase 1b(i). $L_{1} \cong \mathbb{A}_{5}$.

If $E \in \mathcal{F}$, then $L_{1} E \cong \mathbb{S}_{5}$, which has $(Q \mathcal{D})_{2}$. Fix $E \in \mathcal{F}$ with $O_{2}\left(C_{G}\left(L_{1} E\right)\right)=1$ and take $A \in \mathcal{A}_{2}\left(L_{1} E\right)$ exhibiting $(\mathcal{Q D})_{2}$ for $L_{1} E$. Then $L_{1} E=L_{1} A$ and $O_{2}\left(C_{G}\left(L_{1} A\right)\right)=O_{2}\left(C_{G}\left(L_{1} E\right)\right)=1$. The hypotheses of Lemma 3.14 can be checked with $X=\mathcal{A}_{2}(G), H=L_{1} E, K=C_{G}\left(L_{1} E\right)$ and $A$ exhibiting $(Q \mathcal{D})_{2}$ for $L_{1} E$, so $\tilde{H}_{*}\left(\mathcal{A}_{2}(G), \mathbb{Q}\right) \neq 0$. This finishes the proof of subcase $1 \mathrm{~b}(\mathrm{i})$.

Subcase 1b(ii). $L_{1} \cong \mathrm{U}_{3}\left(2^{2}\right)$.

By subcase $1 \mathrm{~b}(\mathrm{i})$, we may also suppose that $L_{2} \neq \mathbb{A}_{5}$. By case 0 and (H2), we may assume that some involution $f \in G$ induces outer automorphisms on $L_{1}$ and $L_{2}$ simultaneously. Since $C_{L_{1}}(f)$ has 2-rank 2, we conclude that $C_{L_{2}}(f)$ has 2-rank 1. This forces $L_{2} \cong \mathrm{L}_{2}(q)$, with $q \geq 5$ odd and $f$ inducing diagonal automorphisms on $L_{2}$, by the classification of simple groups of 2-rank 2 [3, Theorem 48.1]. Moreover, if $\phi \in G$ is a field automorphism of $L_{2}$, then $C_{L_{2}}(\phi) \cong \mathrm{L}_{2}\left(q^{1 / 2}\right)$ has 2-rank 2, which leads 
to $m_{2}\left(\left(L_{1} L_{2}\right)\langle\phi\rangle\right)=5$. This contradicts our main hypothesis that $m_{p}(G)=4$. In conclusion, $G$ does not contain field automorphisms of $L_{2}$, and therefore $G \leq \operatorname{Aut}\left(L_{1}\right) \times \operatorname{Inn} \operatorname{diag}\left(L_{2}\right)$.

By Theorem A.1, $\mathcal{A}_{2}\left(L_{2}\right)$ is connected. That is, $L_{2}$ has $(Q \mathcal{D})_{2}$ exhibited by some $A \in \mathcal{A}_{2}\left(L_{2}\right)$ (of 2-rank 2). Then $O_{2}\left(C_{G}\left(L_{2} A\right)\right)=O_{2}\left(C_{G}\left(L_{2}\right)\right)=1$ by Remark 5.2, and if $B \in \mathcal{A}_{2}(G)_{>A}$, then $B / C_{B}\left(L_{2}\right) \leq \operatorname{Inn} \operatorname{diag}\left(L_{2}\right)$, which has 2-rank 2. Hence $C_{B}\left(L_{2}\right) \neq 1$ and $B=A C_{B}\left(L_{2}\right)$. By Lemma 3.14 applied to $X=\mathcal{A}_{2}(G), H=L_{2}$ and $K=C_{G}\left(L_{2}\right)$, we get $\tilde{H}_{*}\left(\mathcal{A}_{2}(G), \mathbb{Q}\right) \neq 0$. This finishes the proof of subcase $1 \mathrm{~b}(\mathrm{ii})$, and hence of case $1 \mathrm{~b}$ and of case 1 .

Case 2. $p=3$ and $L_{1} \cong \mathrm{L}_{3}\left(2^{2}\right)$.

Note that Out $\left(\mathrm{L}_{3}\left(2^{2}\right)\right) \cong D_{12} \cong C_{3} \rtimes\left(C_{2} \times C_{2}\right)$ and Inn diag $\left(\mathrm{L}_{3}\left(2^{2}\right)\right) \cong \mathrm{L}_{3}\left(2^{2}\right) \rtimes C_{3}$, so without loss of generality, $G \leq \operatorname{Inn} \operatorname{diag}\left(\mathrm{L}_{3}\left(2^{2}\right)\right) \times \operatorname{Aut}\left(L_{2}\right)$. By Proposition 2.3 and the almost-simple case of the conjecture, we can suppose that $G$ is not a direct product of almost-simple groups. Hence, there exists $C \in \mathcal{A}_{3}(G)-\mathcal{A}_{3}\left(L_{1} L_{2}\right)$ of order 3 inducing diagonal automorphisms on $L_{1} \cong \mathrm{L}_{3}\left(2^{2}\right)$. Note that $L_{1} C \cong\left(L_{1} L_{2}\right) C / L_{2} \cong \operatorname{Inn} \operatorname{diag}\left(\mathrm{L}_{3}\left(2^{2}\right)\right)$.

We show that $C$ necessarily induces outer automorphisms on $L_{2}$. If $C$ does not induce outer automorphisms on $L_{2}$, then $C \leq L_{2} C_{G}\left(L_{2}\right)$ and $G$ contains the normal subgroup Inn $\operatorname{diag}\left(L_{3}\left(2^{2}\right)\right)$. This implies that $C_{G}\left(L_{2}\right)=\operatorname{Inn} \operatorname{diag}\left(\mathrm{L}_{3}\left(2^{2}\right)\right)$, so $G$ is the direct product of Inn $\operatorname{diag}\left(\mathrm{L}_{3}\left(2^{2}\right)\right)$ by some almost-simple group $T \leq \operatorname{Aut}\left(L_{2}\right)$ with $F^{*}(T)=L_{2}$. This is a contradiction of our earlier statement that $G$ is not a direct product. Therefore $C$ also induces outer automorphisms on $L_{2}$.

Recall that $C_{L_{3}\left(2^{2}\right)}(C) \cong \mathbb{A}_{5}$ or $C_{7} \rtimes C_{3}$, both of 3-rank 1. By Table 1, the poset $\mathcal{A}_{3}\left(\operatorname{Inn} \operatorname{diag}\left(\mathrm{L}_{3}\left(2^{2}\right)\right)\right)$ is connected (not simply connected) and has dimension 1 . Therefore, there exists $D \in \mathcal{A}_{3}\left(L_{1} C\right)$ of 3rank 2 exhibiting $(Q \mathcal{D})_{3}$ for $L_{1} C$. Note that $L_{1} C=L_{1} D$. If $O_{3}\left(C_{G}\left(L_{1} C\right)\right)=1$ for some $C$, then $\tilde{H}_{*}\left(\mathcal{A}_{3}(G), \mathbb{Q}\right) \neq 0$ by Lemma 3.14 with $X=\mathcal{A}_{3}(G), H=L_{1} D$ and $K=C_{G}\left(L_{1} D\right)=C_{G}\left(L_{1} C\right)$. Thus we can assume that $O_{3}\left(C_{G}\left(L_{1} C\right)\right) \neq 1$ for all $C$. In this case, let $H=L_{1} C_{G}\left(L_{1}\right)$ and $\mathcal{N}=\mathcal{N}(H)$. The subposet $\mathcal{F}:=\mathcal{A}_{3}(G)-\mathcal{N}$ consists of order 3 subgroups acting by diagonal automorphisms on $L_{1} \cong \mathrm{L}_{3}\left(2^{2}\right)$. By Lemma $2.9, \mathcal{A}_{3}(G) \simeq \mathcal{A}_{3}(H)$, so $\tilde{H}_{*}\left(\mathcal{A}_{3}(G), \mathbb{Q}\right)=\tilde{H}_{*}\left(\mathcal{A}_{3}(H), \mathbb{Q}\right) \neq 0$ by $(\mathrm{H} 1)_{\mathbb{Q}}$.

This concludes the proof of the $p$-rank 4 case.

\section{Appendix A. Groups with a strongly $p$-embedded subgroup}

In this appendix we summarise some of the main results on the classification of the groups with a strongly $p$-embedded subgroup, so that the reader can consult them directly here. For further details see $[2,3,8]$.

Recall that a finite group $G$ has a strongly p-embedded subgroup if there exists a proper subgroup $M<G$ such that $M$ contains a Sylow $p$-subgroup of $G$ and $M \cap M^{g}$ is a $p^{\prime}$-group for all $g \in G-M$. By [16, Proposition 5.2], $G$ has a strongly $p$-embedded subgroup if and only if $\mathcal{A}_{p}(G)$ is disconnected. In the following theorem we state the classification of the groups with this property.

Theorem A.1 ([2, (6.2)]). The finite group $G$ has a strongly p-embedded subgroup (i.e., $\mathcal{A}_{p}(G)$ is disconnected) if and only if either $O_{p}(G)=1$ and $m_{p}(G)=1$, or $\Omega_{1}(G) / O_{p^{\prime}}\left(\Omega_{1}(G)\right)$ is one of the following groups:

1. simple of Lie type of Lie rank 1 and characteristic $p$;

2. $\mathbb{A}_{2 p}$ with $p \geq 5$;

3. Aut $\left(\mathrm{L}_{2}\left(2^{3}\right)\right), \mathrm{L}_{3}\left(2^{2}\right)$ or $M_{11}$ with $p=3$;

4. Aut $\left(\mathrm{Sz}\left(2^{5}\right)\right),{ }^{2} F_{4}(2)^{\prime}, M c L$ or Fi $i_{22}$ with $p=5$;

5. $J_{4}$ with $p=11$.

In Table 1 we summarise some properties on the almost-simple groups listed in Theorem A.1. For more details on these assertions, see [8, Part I, sections 7, 9 and 10]. 
Table 1. Properties of almost-simple groups with a strongly p-embedded subgroup.

\begin{tabular}{|c|c|c|c|}
\hline Group $G$ & $\operatorname{Out}(G)$ & $m_{p}(G)$ & $m_{p}(\operatorname{Out}(G))$ \\
\hline \multicolumn{4}{|c|}{$p$-rank 1 almost-simple groups } \\
\hline$G$ & cyclic Sylow $p$-subgroups & 1 & $\leq 1$ \\
\hline \multicolumn{4}{|c|}{ Lie type of Lie rank 1 in characteristic $p$} \\
\hline $\mathrm{L}_{2}\left(p^{a}\right)$ & $C_{\operatorname{gcd}\left(2, p^{a}-1\right)} \rtimes C_{a}$ & $a$ & $m_{p}\left(C_{a}\right) \leq 1$ \\
\hline $\mathrm{U}_{3}\left(p^{a}\right)$ & $C_{\mathrm{gcd}\left(3, p^{a}+1\right)} \rtimes C_{2 a}$ & $\begin{cases}a, & p=2 \\
2 a, & p \neq 2\end{cases}$ & $m_{p}\left(C_{2 a}\right) \leq 1$ \\
\hline $\mathrm{Sz}\left(2^{a}\right), a \geq 3$ odd & $C_{a}$ & $a$ & 0 \\
\hline $\operatorname{Ree}\left(3^{a}\right), a \geq 3$ odd & $C_{a}$ & $2 a$ & $m_{p}\left(C_{a}\right) \leq 1$ \\
\hline \multicolumn{4}{|c|}{ Alternating groups, $p \geq 5$} \\
\hline $\mathbb{A}_{2 p}$ & $C_{2}$ & 2 & 0 \\
\hline \multicolumn{4}{|c|}{$p=3$ exceptions } \\
\hline $\operatorname{Aut}\left(\mathrm{L}_{2}\left(2^{3}\right)\right)$ & 1 & 2 & 0 \\
\hline $\mathrm{L}_{3}\left(2^{2}\right)$ & $D_{12}$ & 2 & 1 \\
\hline$M_{11}$ & 1 & 2 & 0 \\
\hline \multicolumn{4}{|c|}{$p=5$ exceptions } \\
\hline Aut $\left(\mathrm{Sz}\left(2^{5}\right)\right)$ & 1 & 2 & 0 \\
\hline${ }^{2} F_{4}(2)$ & $C_{2}$ & 2 & 0 \\
\hline $\mathrm{McL}$ & $C_{2}$ & 2 & 0 \\
\hline $\mathrm{Fi}_{22}$ & $C_{2}$ & 2 & 0 \\
\hline \multicolumn{4}{|c|}{$p=11$ exception } \\
\hline$J_{4}$ & 1 & 1 & 0 \\
\hline
\end{tabular}

Acknowledgement. I would like to thank Elías Gabriel Minian for his valuable suggestions that helped me to improve the presentation of this article. I am also very grateful to Stephen D. Smith for his careful reading of this article and all of his generous and constructive comments, which significantly improved the presentation, and in particular for many suggestions on the format of section 3, Theorem 1 and the consequences of these methods. This work was supported by a CONICET postdoctoral fellowship, grant PIP 11220170100357, grant PICT 2017-2997 and grant UBACYT 20020160100081BA.

Conflict of Interest: None.

\section{References}

[1] J. L. Alperin, 'A Lie approach to finite groups', in Groups-Canberra 1989, Lecture Notes in Mathematics, vol. 1456 (Springer, Berlin, 1990), 1-9.

[2] M. Aschbacher, 'Simple connectivity of p-group complexes', Israel J. Math. 82(1-3) (1993), 1-43.

[3] M. Aschbacher, Finite Group Theory, second edn, Cambridge Studies in Advanced Mathematics, vol. 10 (Cambridge University Press, Cambridge, UK, 2000).

[4] M. Aschbacher and P.B. Kleidman, 'On a conjecture of Quillen and a lemma of Robinson', Arch. Math. (Basel) 55(3) (1990), 209-217.

[5] M. Aschbacher and S. D. Smith, 'On Quillen's conjecture for the p-groups complex', Ann. of Math. (2) 137(3) (1993), 473-529.

[6] K. S. Brown, 'Euler characteristics of groups: the $p$-fractional part', Invent. Math. 29(1) (1975), 1-5.

[7] A. Díaz Ramos, 'On Quillen's conjecture for $p$-solvable groups', J. Algebra 513 (2018), 246-264.

[8] D. Gorenstein and R. Lyons, 'The local structure of finite groups of characteristic 2 type', Mem. Amer. Math. Soc. 42(276) (1983).

[9] J. Grodal, 'Higher limits via subgroup complexes', Ann. of Math. (2) 155(2) (2002), 405-457.

[10] M. W. Jacobsen and J. M. Møller, 'Euler characteristics and Möbius algebras of $p$-subgroup categories', J. Pure Appl. Algebra 216(12) (2012), 2665-2696.

[11] E. G. Minian and K. I. Piterman, 'The homotopy types of the posets of $p$-subgroups of a finite group', Adv. Math. 328 (2018), 1217-1233. 
[12] E. G. Minian and K. I. Piterman, 'The fundamental group of the p-subgroup complex', J. Lond. Math. Soc. (2) 103 (2021), 449-469.

[13] K. I. Piterman, 'A stronger reformulation of Webb's conjecture in terms of finite topological spaces', J. Algebra 527 (2019), 280-305.

[14] K. I. Piterman, I. Sadofschi Costa and A. Viruel, 'Acyclic 2-dimensional complexes and Quillen's conjecture', Publ. Mat. 65 (2021), 129-140.

[15] J. Pulkus and V. Welker, 'On the homotopy type of the $p$-subgroup complex for finite solvable groups', J. Aust. Math. Soc. Ser. A 69(2) (2000), 212-228.

[16] D. Quillen, 'Homotopy properties of the poset of nontrivial p-subgroups of a group', Adv. Math. 28(2) (1978), 101-128.

[17] G. R. Robinson, 'Some remarks on permutation modules', J. Algebra 118 (1988), 46-62.

[18] Y. Segev, 'Quillen's conjecture and the kernel on components', Comm. Algebra 24(3) (1996), 955-962.

[19] Y. Segev and P. Webb, 'Extensions of G-posets and Quillen's complex', J. Aust. Math. Soc. Ser. A 57(1) (1994), 60-75.

[20] S.D. Smith, Subgroup Complexes, Mathematical Surveys and Monographs, vol. 179 (American Mathematical Society, Providence, RI, 2011). 